\title{
Stability in Nonautonomous Dynamics: A Survey of Recent Results
}

\section{Luis Barreira}

Departamento de Matemática, Instituto Superior Técnico, 1049-001 Lisboa, Portugal

E-mail address: barreira@math.ist.utl.pt

$U R L:$ http: //www.math.ist.utl.pt/ barreira

\section{Claudia Vall}

Departamento de Matemática, Instituto Superior Técnico, 1049-001 Lisboa, Portugal

E-mail address: cvalls@math.ist.utl.pt

\begin{abstract}
We discuss recent results in the stability theory of nonautonomous differential equations under sufficiently small perturbations. We mostly concentrate on the description of our own work, with emphasis on the Lyapunov stability of solutions, the existence and smoothness of invariant manifolds, and the construction and regularity of topological conjugacies, among other topics. The main novelty is that we always consider a nonuniform exponential behavior of the linear variational equations, given either by the existence of a nonuniform exponential contraction or a nonuniform exponential dichotomy.
\end{abstract}

\section{Contents}

1. Introduction

2. Exponential contractions and stability 140

3. Exponential dichotomies 145

4. Stable invariant manifolds 149

5. Construction of conjugacies 158

6. Center manifolds and reversibility 163

Key words: exponential dichotomies, invariant manifolds, stability theory.

Supported by the Center for Mathematical Analysis, Geometry, and Dynamical Systems, and through Fundação para a Ciência e a Tecnologia by Program POCTI/FEDER, Program POSI, and the grant SFRH/BPD/26465/2006. 
7. Lyapunov regularity 166

References

\section{Introduction}

The unifying theme of this survey is the stability of nonautonomous differential equations under sufficiently small nonlinear perturbations, with emphasis on the study of the Lyapunov stability of solutions, and of the existence and smoothness of invariant manifolds. We describe recent results in the area, mostly concentrating on our own work. The main novelty is that we always consider a nonuniform exponential behavior of the linear variational equations. This causes that the stability results discussed in the survey hold for a much larger class of differential equations than in the "classical" theory of exponential dichotomies. In addition, whenever possible, we consider the infinite-dimensional case. In view of the readability of the survey, instead of always presenting the most general results, we made an appropriate selection of the material, although this selection should also reflect a personal taste.

\subsection{Exponential dichotomies and generalizations}

In the theory of differential equations, both in finite-dimensional and infinite-dimensional spaces, the notion of exponential dichotomy, introduced by Perron in [57], plays a central role in the study of the stability of solutions, and in particular in the theory of stable and unstable invariant manifolds. For example, let $u(t)$ be a solution of the equation $u^{\prime}=F(u)$, for some differentiable map $F$ in a Banach space. Setting

$$
A(t)=d_{u(t)} F,
$$

the existence of an exponential dichotomy for the linear variational equation

$$
v^{\prime}=A(t) v,
$$

with some additional assumptions on the nonlinear part of the vector field, guarantees the existence of stable and unstable invariant manifolds for the solution $u(t)$.

We note that the theory of exponential dichotomies and its applications are quite developed. In particular, there exist several classes of linear differential equations with exponential dichotomies. We can mention, for example, the series of papers of Sacker and Sell [71, 72, 73, 70, 74] that in particular discuss sufficient conditions for the existence of exponential dichotomies, also in the infinite-dimensional setting. In a different direction, for example, geodesic flows on compact smooth Riemannian manifolds 
with negative sectional curvature have the whole unit tangent bundle as a hyperbolic set, i.e., they define Anosov flows. For any such flow the linear variational equation of each trajectory has an exponential dichotomy. Furthermore, time changes and small $C^{1}$ perturbations of flows with a hyperbolic set also possess hyperbolic sets (see for example [41]). We refer to the books $[21,35,38,75]$ for details and further references related to exponential dichotomies. We particularly recommend [21] for historical aspects. One may also consult the interesting books $[27,28,50]$, in spite of being less recent. On the other hand, even though all the literature and the intense research activity in the area over the years, the notion of exponential dichotomy considerably restricts the dynamics. It is thus important to look for more general types of hyperbolic behavior.

Our main objective is precisely to consider the more general notion of nonuniform exponential dichotomy and study in a systematic manner some of its consequences, in particular in connection with the existence and the smoothness of invariant manifolds for nonautonomous differential equations. We also discuss a version of the Grobman-Hartman theorem, the existence of center manifolds, as well as their reversibility properties, and Lyapunov's regularity theory and its applications to the stability of solutions of nonautonomous equations. In comparison with the classical notion of (uniform) exponential dichotomy, the existence of a nonuniform exponential dichotomy is a much weaker assumption. In particular, perhaps surprisingly, we will see that in a certain sense any linear equation as in (1), having global solutions and at least one nonzero Lyapunov exponent, admits a nonuniform exponential dichotomy.

\subsection{Nonuniform hyperbolicity}

Our work is also a contribution to the nonuniform hyperbolicity theory, with emphasis on differential equations and with a systematic development in the infinite-dimensional setting. We refer to $[1,3]$ for detailed expositions of parts of the nonuniform hyperbolicity theory and [2] for a detailed description of its contemporary status. The theory goes back to the landmark works of Oseledets [54] and particularly Pesin [58, 59,60]. Since then it became an important part of the general theory of dynamical systems and a principal tool in the study of stochastic behavior. We note that the nonuniform hyperbolicity conditions can be expressed in terms of the Lyapunov exponents. For example, almost all trajectories of a dynamical system preserving a finite invariant measure with nonzero Lyapunov exponents are nonuniformly hyperbolic.

Among the most important properties due to nonuniform hyperbolicity is the existence of stable and unstable invariant manifolds, and their absolute continuity property established by Pesin in [58]. The theory also describes 
the ergodic properties of dynamical systems with a finite invariant measure that is absolutely continuous with respect to the volume [59], and expresses the Kolmogorov-Sinai entropy in terms of the Lyapunov exponents by the Pesin entropy formula [59] (see also [45]). In another direction, combining the nonuniform hyperbolicity with the nontrivial recurrence guaranteed by the existence of a finite invariant measure, the fundamental work of Katok [40] revealed a very rich and complicated orbit structure, including an exponential growth rate for the number of periodic points measured by the topological entropy, and the approximation of the entropy of an invariant measure by uniformly hyperbolic horseshoes (see also [3]).

We now consider the particular case of the stable manifold theorem, and we compare the existing results with our own work. We first briefly describe the relevant references. The proof by Pesin in [58] is an elaboration of the classical work of Perron. In [68] Ruelle obtained a proof of the stable manifold theorem based on the study of perturbations of products of matrices in Oseledets' multiplicative ergodic theorem [54]. Another proof is due to Pugh and Shub in [66] with an elaboration of the classical work of Hadamard using graph transform techniques. In [31] Fathi, Herman and Yoccoz provided a detailed exposition of the stable manifold theorem essentially following the approaches of Pesin and Ruelle. There are also versions of the stable manifold theorem for dynamical systems in infinitedimensional spaces. In [69] Ruelle established a version in Hilbert spaces, following his approach in [68]. In [48] Mañé considered transformations in Banach spaces under certain compactness and invertibility assumptions, including the case of differentiable maps with compact derivative at each point. On the other hand, in [65] Pugh constructed a $C^{1}$ diffeomorphism in a manifold of dimension 4, that is not of class $C^{1+\varepsilon}$ for any $\varepsilon$, and for which there exists no invariant manifold tangent to a certain stable space such that the trajectories along the invariant manifold travel with exponential speed. But this example does not forbid the existence of a $C^{1}$ dynamics, which is not of class $C^{1+\varepsilon}$ for any $\varepsilon$, for which there still exist stable manifolds. We show in $[5,7]$ that there exist stable invariant manifolds for the nonuniformly hyperbolic trajectories of a large family of dynamics that, in general, are at most of class $C^{1}$. Furthermore, in [9] we obtain $C^{k}$ stable manifolds in Banach spaces, using a single fixed point problem to obtain all derivatives simultaneously.

\subsection{Uniform contractions and nonuniform contractions}

In order to describe the main problems in which we are interested, we first introduce the notions of uniform contraction and nonuniform contraction. We note that these are simpler notions than those of (uniform) exponential dichotomy and nonuniform exponential dichotomy. On the other hand, 
this simplification allows us to describe the main ideas without accessory technicalities.

Consider the linear equation (1) in a Banach space $X$, for a family of bounded linear operators $A(t)$ varying continuously with $t \geq 0$. We assume that (1) has unique solutions that are defined for all time $t \geq 0$. We also consider the equation

$$
v^{\prime}=A(t) v+f(t, v)
$$

for a continuous function $f$ such that $f(t, 0)=0$ for every $t \geq 0$. In particular, $v(t)=0$ is still a solution of equation (2).

When there exist constants $c>0$ and $\lambda<0$ such that

$$
\|v(t)\| \leq c e^{\lambda(t-s)}\|v(s)\|, \quad t \geq s
$$

for every solution $v(t)$ of equation (1), we say that (1) admits a uniform exponential contraction. One can then show that for each "sufficiently small" perturbation $f$, the zero solution of equation (2) is uniformly asymptotically stable and in fact uniformly exponentially stable. This follows from a simple argument using the variation-of-constants formula to obtain a fixed point problem, and then showing that this problem has a unique solution in a certain space of functions with exponential decay.

The situation changes drastically if condition (3) is replaced by the weaker assumption that all Lyapunov exponents of equation (1) are negative, i.e., that

$$
\limsup _{t \rightarrow+\infty} \frac{1}{t} \log \|v(t)\|<0
$$

for any solution $v(t)$ of equation (1). Indeed, in this situation there may exist arbitrarily small perturbations $f$ such that the zero solution of equation (2) is not asymptotically stable, as the following example shows.

Example 1.1 (see [1, Example 1.1.1]). We consider the linear equation in $\mathbb{R}^{2}$ with matrix of coefficients

$$
A(t)=\left(\begin{array}{cc}
-15-14 a(t) & 0 \\
0 & -15+14 a(t)
\end{array}\right)
$$

defined for $t>0$, where

$$
a(t)=\sin \log t+\cos \log t .
$$

For example, for the perturbation

$$
f(t,(x, y))=\left(0, x^{4}\right),
$$

one can show that although the limsup in (4) is constant and equal to -1 , there exist solutions $v(t)$ of equation (2) for which

$$
\limsup _{t \rightarrow+\infty} \frac{1}{t} \log \|v(t)\|>0
$$


(we refer to [1] for details). In other words, assuming that all Lyapunov exponents are negative is not sufficient to guarantee that the asymptotic stability of the zero solution of equation (1) persists under sufficiently small nonlinear perturbations.

We now briefly explain the difficulty in establishing the asymptotic stability in equation (2) when the ambient space $X$ is finite-dimensional. Assume that condition (4) holds, i.e., that all Lyapunov exponents are negative. Since the space of solutions of equation (1) is finite-dimensional (since it has the same dimension as $X$ ), one can easily show that there exist constants $D>0, \lambda<0$ and $\varepsilon \geq 0$ such that

$$
\|v(t)\| \leq D e^{\lambda(t-s)+\varepsilon s}\|v(s)\|, \quad t \geq s .
$$

When this happens we say that equation (1) admits a nonuniform exponential contraction. We emphasize that one is not able to deduce from (4) that $\varepsilon=0$. When $\varepsilon>0$ the estimate in (5) can be shown to imply that the size of the neighborhood at time $s$ where the exponential stability of the zero solution of equation (2) is guaranteed decreases with exponential rate at least $\varepsilon$ (see Section 2.2). This is due to the fact that when we perturb equation (1) the exponential term $e^{\varepsilon s}$ in (5) may amplify the perturbation, and thus to make sure that this is not the case we must take the initial condition $v(s)$ so that $e^{\varepsilon s}\|v(s)\|$ is sufficiently small (that is, $v(s)$ must be approximately of size $\left.e^{-\varepsilon s}\right)$. We refer to Section 2.2 for rigorous statements.

\subsection{Formulation of the main problems}

The former discussion also motivates some of the main problems that we discuss in the survey. We now briefly describe these problems.

\section{Persistence of asymptotic stability}

The study of asymptotic stability is at the center of our discussion. Thus, one of the main problems is to find conditions that guarantee the persistence of asymptotic stability in equation (2), assuming that equation (1) admits a nonuniform exponential contraction. We describe sufficient conditions that are much weaker than the uniform asymptotic stability exhibited by a uniform exponential contraction. Furthermore, we also consider the situation when the constant $\varepsilon$ in (5) is positive and cannot be made arbitrarily small.

\section{Exponential dichotomies}

We also want to consider the case when the solutions of equation (1) exhibit not only contraction but also expansion. In this case the notion of stability is replaced by the asymptotic stability along an invariant manifold, that is tangent to the space containing the solutions of (1) with negative 
Lyapunov exponent. In particular, we describe the construction of stable (and unstable) invariant manifolds, in the infinite-dimensional setting. We also study their regularity, with a single fixed point problem to obtain all derivatives simultaneously.

\section{Robustness of nonuniform exponential behavior}

Besides the problem of persistence of asymptotic stability in equation (1) under nonlinear perturbations it is also important to know whether the stability persists under sufficiently small linear perturbations. This is the so-called robustness problem. It corresponds to consider the linear equation

$$
v^{\prime}=[A(t)+B(t)] v
$$

and ask whether it admits a nonuniform exponential contraction (or a nonuniform exponential dichotomy), for a sufficiently small perturbation $B$, when the same happens with the original equation (1). We give conditions for the robustness of the nonuniform exponential behavior. We also discuss how the constants $\lambda$ and $\varepsilon$ in the notion of exponential contraction vary with the perturbation.

\section{Estimates for the constants determining the hyperbolicity}

In the study of the persistence of asymptotic stability, the conditions determining the allowed perturbations are expressed in terms of the constants $\lambda$ and $\varepsilon$. Thus, it is important to obtain sharp estimates for these constants, preferably in terms of the operators $A(t)$, without the need to solve explicitly equation (1). We describe estimates for a large class of equations. We recall that there are examples, even within Floquet theory, with all matrices $A(t)$ having only eigenvalues with negative real part, but for which there exists a positive Lyapunov exponent. This shows once more that the study of the asymptotic behavior of solutions of equation (1) is very delicate.

\section{Construction of topological conjugacies}

A fundamental problem in the study of the local behavior of a dynamical system is whether the linearization of the dynamics along a given solution approximates well the solution. In other words, we look for a local change of variables, called a conjugacy, that takes the system to a linear one. Moreover, to distinguish the dynamics in a neighborhood of the solution further than in the topological category, the change of variables must be as regular as possible. We discuss this problem in the general setting of nonuniform hyperbolicity, with a version of the Grobman-Hartman theorem. In addition we show that the (topological) conjugacies are always Hölder continuous. 


\section{Central behavior}

It is also important to allow central directions, in which there is neither contraction nor expansion, at least with exponential speed, and obtain corresponding results concerning the persistence of the central behavior in equation (1) under sufficiently small nonlinear perturbations. This corresponds to establish the existence of invariant center manifolds for equation (2). As is well known, these are powerful tools in the analysis of the asymptotic behavior of dynamical systems. For example, when equation (1) possesses no unstable directions, the stability of the origin in equation (2) can be determined by studying the behavior on (any) center manifold.

\section{Speeds of nonuniform exponential behavior and optimal estimates}

There are many examples of contractions for which the constant $\varepsilon$ in (5) cannot be taken equal to zero. On the other hand, in many of these examples one can replace the term $D e^{\varepsilon s}$ in (5) by a function $D(s)$ with subexponential growth, i.e., such that

$$
\limsup _{s \rightarrow+\infty} \frac{1}{s} \log D(s)=0 .
$$

In this case, some of the results presented in the survey can be improved. For example, it is possible to obtain optimal estimates for the decay of solutions of the perturbed equation (2) expressed in terms of $D(s)$ and of the constant $\lambda$ in (5). This is a more technical problem, although the required changes are not tremendous. We do not discuss any related matter in the survey and we refer to [16] for full details.

\section{Exponential contractions and stability}

This section is dedicated to the study of the persistence of asymptotic stability of a nonuniform exponential contraction under a sufficiently small nonlinear perturbation. Instead of starting the discussion with the more general notion of nonuniform exponential dichotomy, we start with the notion of contraction which allows us to describe the main ideas without accessory technicalities.

We first give nontrivial examples of nonuniform contractions, in the sense that they are not uniform, exhibiting different speeds of nonuniform behavior. We then discuss the persistence of asymptotic stability of nonuniform exponential contractions under perturbations. We also give sharp estimates for the constants in the notion of nonuniform contraction. Finally, we discuss the existence of nonuniform contractions at large, in particular in connection with ergodic theory. 


\subsection{Nonuniform exponential contractions}

Let $B(X)$ be the set of bounded linear operators in the Banach space $X$. We consider a continuous function $A: \mathbb{R}_{0}^{+} \rightarrow B(X)$, and the initial value problem

$$
v^{\prime}=A(t) v, \quad v(s)=v_{s},
$$

with $s \geq 0$ and $v_{s} \in X$. As in Section 1.3, we always assume that all solutions of equation (7) are global, i.e., that

$$
\text { each solution of (7) is defined for every } t \geq 0 \text {. }
$$

We denote by $T(t, s)$ the linear operator such that

$$
T(t, s) v(s)=v(t)
$$

for each solution $v$ of equation (7) and each $t, s \geq 0$.

Definition 2.1. We say that equation (7) admits a nonuniform exponential contraction if there exist constants $D>0, \lambda<0$, and $\varepsilon \geq 0$ such that

$$
\|T(t, s)\| \leq D e^{\lambda(t-s)+\varepsilon s}, \quad t \geq s \geq 0 .
$$

When one can take $\varepsilon=0$ in (10) we also say that equation (7) admits a uniform exponential contraction (and thus a uniform contraction is a particular case of a nonuniform one).

We now give examples of nonuniform exponential contractions that are not uniform.

Example 2.2 ([16, Example 1]). For $\lambda<0$ and $a>0$, the equation in $\mathbb{R}$ given by

$$
v^{\prime}=\left(\lambda+\frac{a}{t+1}(\cos t-1)-a \log (t+1) \sin t\right) v
$$

(for $t>-1$ ) admits a nonuniform exponential contraction. Indeed, we have

$$
T(t, s)=e^{\lambda(t-s)+a \log (t+1)(\cos t-1)-a \log (s+1)(\cos s-1)}
$$

(that is, the linear operator $T(t, s)$ coincides with multiplication by the exponential in (12)), which implies that

$$
T(t, s) \leq(s+1)^{2 a} e^{\lambda(t-s)}, \quad t \geq s \geq 0 .
$$

Therefore, for each $\varepsilon>0$ there exists $D>0$ such that inequality (10) holds. In particular, equation (11) admits a nonuniform exponential contraction. We note that one is not able to take $\varepsilon=0$ in inequality (10): for example, taking $t=2 k \pi$ and $s=(2 l-1) \pi$ with $k, l \in \mathbb{N}$ we find that

$$
T(t, s)=(s+1)^{2 a} e^{\lambda(t-s)},
$$

and thus the estimate in (13) cannot be improved. 
We now give an example of nonuniform exponential contraction for which the exponential term $e^{\varepsilon s}$ in (10) cannot be improved.

Example 2.3 ([12, Proposition 3]). For $\lambda<a<0$, the equation in $\mathbb{R}$ given by $v^{\prime}=(\lambda+a t \sin t) v$ admits a nonuniform exponential contraction. We have

$$
\begin{aligned}
T(t, s) & =e^{\lambda(t-s)-a t \cos t+a \sin t+a s \cos s-a \sin s} \\
& =e^{(\lambda-a)(t-s)-a t(\cos t-1)+a s(\cos s-1)+a(\sin t-\sin s)} .
\end{aligned}
$$

It follows that

$$
T(t, s) \leq e^{2|a|} e^{(\lambda-a)(t-s)+2|a| s}, \quad t \geq s \geq 0,
$$

and inequality (10) holds with the constants $D=e^{2|a|}$ and $\varepsilon=2|a|>0$. Furthermore, for $t=2 k \pi$ and $s=(2 l-1) \pi$ with $k, l \in \mathbb{N}$ we have

$$
T(t, s)=e^{(\lambda-a)(t-s)+2|a| s},
$$

and thus the term $2|a| s$ in (14) is as small as possible.

\subsection{Stability of nonuniform exponential contractions}

We address in this section the problem of the persistence of asymptotic stability, under sufficiently small perturbations of equation (7).

We consider perturbations of equation (7) given by a continuous function $f: \mathbb{R}_{0}^{+} \times X \rightarrow X$ such that

$$
f(t, 0)=0 \text { for every } t \geq 0,
$$

and we assume that there exist constants $c>0$ and $q>0$ such that

$$
\|f(t, u)-f(t, v)\| \leq c\|u-v\|\left(\|u\|^{q}+\|v\|^{q}\right)
$$

for every $t \geq 0$ and $u, v \in X$. We consider the initial value problem

$$
v^{\prime}=A(t) v+f(t, v), \quad v(s)=v_{s},
$$

with $s \geq 0$ and $v_{s} \in X$. We note that by condition (15) the function $v(t)=0$ is still a solution of equation (17).

The following result establishes the persistence of asymptotic stability of nonuniform exponential contractions under sufficiently small perturbations. We denote by $B(\delta) \subset X$ the open ball of radius $\delta>0$ centered at zero, and we set $\alpha=\varepsilon(1+1 / q)$.

Theorem 2.4. If equation (7) admits a nonuniform exponential contraction with $q \lambda+\varepsilon<0$, then for each $K>D$ there exists $\delta>0$ such that for every $s \geq 0, v_{s}, \bar{v}_{s} \in B\left(\delta e^{-\alpha s}\right)$ and $t \geq s$ we have

$$
\|v(t)-\bar{v}(t)\| \leq K e^{\lambda(t-s)+\varepsilon s}\left\|v_{s}-\bar{v}_{s}\right\|,
$$

where $v(t)$ and $\bar{v}(t)$ are the solutions of (17) with $v(s)=v_{s}$ and $\bar{v}(s)=\bar{v}_{s}$. 
Theorem 2.4 is a particular case of Theorem 1 in [16]. Notice that we need to take the initial conditions $v_{s}$ and $\bar{v}_{s}$ in a neighborhood of size $\delta e^{-\alpha s}$. Essentially, this ensures that the exponential term $e^{\varepsilon s}$ in (10) is not amplified in equation (17).

When $\varepsilon=0$ we recover the following classical result.

Theorem 2.5. If equation (7) admits a uniform exponential contraction, then for each $K>D$ there exists $\delta>0$ such that for every $s \geq 0, v_{s}, \bar{v}_{s} \in$ $B(\delta)$ and $t \geq s$ we have

$$
\|v(t)-\bar{v}(t)\| \leq K e^{\lambda(t-s)}\left\|v_{s}-\bar{v}_{s}\right\| .
$$

The main differences between Theorems 2.4 and 2.5 are that in the second theorem the condition $q \lambda+\varepsilon<0$ is automatically satisfied, and that the neighborhoods $B\left(\delta e^{-\alpha s}\right)$ are replaced by the single neighborhood $B(\delta)$, which is independent of $s$.

\subsection{Existence of nonuniform exponential contractions}

It should be emphasized that the existence of a uniform exponential contraction (see (3)), is a very restrictive assumption. On the other hand, the existence of a nonuniform exponential contraction (see (10)) is a more typical situation, although not only because it is a weaker assumption. To make this statement more precise we formulate two theorems which indicate that nonuniform exponential contractions are very common, at least in finite-dimensional spaces. We emphasize that Sections 2.3 and 2.4 consider only the finite-dimensional case. This is due to the fact that the theory is not yet developed for arbitrary Banach spaces. We refer to [6] for an appropriate generalization in Hilbert spaces.

The fact that condition (4) implies condition (10) can be reformulated in the following manner.

Theorem 2.6 ([12, Theorem 1$])$. Let $A(t)$ be $n \times n$ matrices varying continuously with $t$. If condition (4) holds then equation (1) admits a nonuniform exponential contraction.

In particular, Theorem 2.6 indicates that the constants $\lambda$ and $\varepsilon$ in (10) occur naturally in the study of asymptotic stability. Furthermore, the theorem shows that in a certain sense the notion of nonuniform exponential contraction is the weakest possible notion of exponential contraction. Thus, one can argue that the approach presented in the survey is as general as possible, although sometimes with weaker statements.

On the other hand, in view of the condition $q \lambda+\varepsilon<0$ in Theorem 2.4, for a given $\lambda$ either the order $q$ of the perturbation $f$ is sufficiently large or the constant $\varepsilon$ is sufficiently small. It turns out, at least from the point of 
view of ergodic theory, that $\varepsilon$ can be made arbitrarily small almost always. To formulate a rigorous statement we recall that a flow $\Psi_{t}: \mathbb{R}^{n} \rightarrow \mathbb{R}^{n}$ is said to preserve a measure $\mu$ in $\mathbb{R}^{n}$ if

$$
\mu\left(\Psi_{t} A\right)=\mu(A) \text { for every measurable set } A \subset \mathbb{R}^{n} \text { and every } t \in \mathbb{R} .
$$

Theorem 2.7. Let $F: \mathbb{R}^{n} \rightarrow \mathbb{R}^{n}$ be a vector field of class $C^{1}$ determining a flow $\Psi_{t}$ that preserves a finite measure $\mu$ in $\mathbb{R}^{n}$. For $\mu$-almost every $x \in \mathbb{R}^{n}$, if condition (4) holds for equation (1) with $A(t)=d_{\Psi_{t} x} F$, then the equation admits a nonuniform exponential contraction with arbitrarily small $\varepsilon>0$.

Theorem 2.7 is a simple consequence of Oseledets' multiplicative ergodic theorem in [54] (see for example [3, Section 3.4] for a detailed discussion). It shows that in the context of ergodic theory the nonuniformity given by the constant $\varepsilon$ in (10) can be made arbitrarily small almost always, although not necessarily zero. Therefore, it is important to study the asymptotic stability in the general nonuniform case.

\subsection{Estimates for the constants}

When $\varepsilon>0$, the condition $q \lambda+\varepsilon<0$ in Theorem 2.4 requires a compromise between the rate of contraction (given by $\lambda$ ) and the order of the perturbation $f$ (given by $q$ ). It is thus important to estimate the constants $\lambda$ and $\varepsilon$ in the notion of nonuniform exponential contraction.

Fortunately, there exists a device, developed by Lyapunov, that allows one to measure the deviation of a nonuniform exponential contraction with respect to a uniform contraction. This is the so-called regularity theory (see Section 7), introduced by Lyapunov in his doctoral thesis [47] in the case of finite dimension. Unfortunately, this theory seems nowadays somewhat overlooked in the context of differential equations. A generalization to infinite dimension is given in [6] although only for Hilbert spaces.

We first formulate a rigorous statement with a sharp estimate for the constant $\lambda$. Consider $n \times n$ matrices $A(t)$ varying continuously with $t$, and assume that condition (8) holds.

Theorem 2.8 ([12, Theorem 1$]$ ). If for every $v_{0} \in \mathbb{R}^{n}$ we have

$$
\lambda\left(v_{0}\right):=\limsup _{t \rightarrow+\infty} \frac{1}{t} \log \|v(t)\|<0,
$$

where $v(t)$ is the solution of equation (7) with $v(0)=v_{0}$, then the equation admits a nonuniform exponential contraction and we can take in (10) any $\lambda<0$ such that

$$
\lambda>\sup _{v_{0} \in \mathbb{R}^{n}} \lambda\left(v_{0}\right)
$$


The function $v_{0} \mapsto \lambda\left(v_{0}\right)$ defined by (18) is called the Lyapunov exponent associated to equation (7) (see also Section 7). We note that the supremum in (19) is indeed negative, since the Lyapunov exponent can take only finitely many values (this follows easily from the fact that the space of solutions of equation (7) is finite-dimensional).

We now consider the constant $\varepsilon$. For simplicity of the exposition, we consider only the case of triangular matrices.

Theorem 2.9 ([12, Theorem 2]). Assume that the matrices $A(t)$ are upper triangular for each $t \geq 0$. If equation (7) admits a nonuniform exponential contraction, then we can take in (10) any $\varepsilon>0$ such that

$$
\varepsilon>\sum_{k=1}^{n}\left(\limsup _{t \rightarrow+\infty} \frac{1}{t} \int_{0}^{t} a_{k}(\tau) d \tau-\liminf _{t \rightarrow+\infty} \frac{1}{t} \int_{0}^{t} a_{k}(\tau) d \tau\right),
$$

where $a_{1}(t), \ldots, a_{n}(t)$ are the entries in the diagonal of $A(t)$.

Theorem 2.9 is a simple consequence of Theorems 7.3 and 7.5, which are based on the regularity theory of Lyapunov. The case of arbitrary matrices can be treated by first making a reduction to the triangular case (see [1, Lemma 1.3.3]). We refer to [12] for details.

\section{Exponential dichotomies}

We now consider the more general case of nonuniform exponential dichotomies. This section is dedicated to the linear theory; we refer to Sections 4-6 for the discussion of the nonlinear theory, with emphasis on the construction of invariant manifolds and topological conjugacies.

In particular we discuss the robustness of nonuniform exponential dichotomies, that is, whether a sufficiently small linear perturbation of a linear equation with a nonuniform exponential dichotomy stills admits such a dichotomy.

\subsection{Nonuniform exponential dichotomies}

We continue to consider a continuous function $A: \mathbb{R}_{0}^{+} \rightarrow B(X)$, and the initial value problem (7) with $s \geq 0$ and $v_{s} \in X$. We assume that (8) holds, and we denote by $T(t, s)$ the linear operator such that (9) holds for each solution $v$ of equation (7) and each $t, s \geq 0$.

Definition 3.1. We say that equation (7) admits a nonuniform exponential dichotomy if there exists a function $P: \mathbb{R}_{0}^{+} \rightarrow B(X)$ such that $P(t)$ is a projection for each $t \geq 0$, with

$$
P(t) T(t, s)=T(t, s) P(s) \text { for every } t, s \geq 0,
$$


and there exist constants

$$
D>0, \quad \bar{a}<0 \leq \underline{b}, \quad \text { and } \quad \varepsilon \geq 0
$$

such that for every $t \geq s \geq 0$ we have

$$
\|T(t, s) P(s)\| \leq D e^{\bar{a}(t-s)+\varepsilon s}, \quad\left\|T(t, s)^{-1} Q(t)\right\| \leq D e^{-\underline{b}(t-s)+\varepsilon t},
$$

where $Q(t)=\mathrm{Id}-P(t)$ is the complementary projection for each $t \geq 0$.

The notion of nonuniform exponential dichotomy mimics the classical notion of (uniform) exponential dichotomy. Recall that equation (7) admits a (uniform) exponential dichotomy if there exist a function $P$ as in Definition 3.1 and constants $D, \beta>0$ such that for every $t \geq s \geq 0$ we have

$$
\|T(t, s) P(s)\| \leq D e^{-\beta(t-s)}, \quad\left\|T(t, s)^{-1} Q(s)\right\| \leq D e^{-\beta(t-s)} .
$$

We note that we allow the constant $\underline{b}$ in $(21)$ to be zero. We emphasize that this is not an essential feature of the notion of nonuniform exponential dichotomy. Incidentally, in the theory of stable invariant manifolds (see Section 4) the inequalities $\bar{a}<0 \leq \underline{b}$ in (21) are the minimal hypotheses in the notion of dichotomy that allow us to construct the manifolds. We could consider instead the situation when $\bar{a} \leq 0<\underline{b}$, and develop an entirely analogous theory to construct invariant unstable manifolds (simply by reversing time). The details are simple and are not discussed in the survey.

We also consider a stronger form of nonuniform exponential dichotomy.

Definition 3.2. We say that equation (7) admits a strong nonuniform exponential dichotomy if there exists a function $P: \mathbb{R}_{0}^{+} \rightarrow B(X)$ such that $P(t)$ is a projection for each $t \geq 0$ and (20) holds, and there exist constants

$$
D \geq 0, \quad \underline{a} \leq \bar{a}<0 \leq \underline{b} \leq \bar{b}, \quad \text { and } \quad \varepsilon \geq 0
$$

such that for every $t \geq s \geq 0$ we have

$$
\begin{array}{lll}
\|T(t, s) P(s)\| \leq D e^{\bar{a}(t-s)+\varepsilon s}, & \left\|T(t, s)^{-1} P(t)\right\| \leq D e^{-\underline{a}(t-s)+\varepsilon t}, \\
\|T(t, s) Q(s)\| \leq D e^{\bar{b}(t-s)+\varepsilon s}, & \left\|T(t, s)^{-1} Q(t)\right\| \leq D e^{-\underline{b}(t-s)+\varepsilon t} .
\end{array}
$$

Clearly, any strong nonuniform exponential dichotomy is also a nonuniform exponential dichotomy. On the other hand, even when $X$ is finitedimensional, a linear equation admitting a nonuniform exponential dichotomy may not admit a strong nonuniform exponential dichotomy, as the following example shows. Consider the equation in $\mathbb{R}^{2}$ given by

$$
x^{\prime}=-x, \quad y^{\prime}=t y .
$$

One can easily verify that (25) admits a nonuniform exponential dichotomy with $\bar{a}=-1, \underline{b}=0, \varepsilon=0$, and $D=1$. Furthermore, the second inequality 
in (24) holds with $\underline{a}=-1$. But we would need to take $\bar{b}=+\infty$ so that the third inequality in (24) could hold, and thus equation (25) does not admit a strong nonuniform exponential dichotomy.

More generally, one can introduce the notion of nonuniform exponential dichotomy for an arbitrary evolution operator $T(t, s)$, without considering an associated linear equation, and thus, in particular, without any a priori assumption on the regularity of the map $(t, s) \mapsto T(t, s)$. We can also introduce the notion of nonuniform exponential dichotomy in an arbitrary interval $J$. In this case, instead of the inequalities in (22) we require that

$$
\|T(t, s) P(s)\| \leq D e^{\bar{a}(t-s)+\varepsilon|s|}, \quad\left\|T(t, s)^{-1} Q(t)\right\| \leq D e^{-\underline{b}(t-s)+\varepsilon|t|}
$$

for every $t, s \in J$ with $t \geq s$. Analogously, we introduce the notion of strong nonuniform exponential dichotomy in an arbitrary interval $J$.

Building on the examples of nonuniform exponential contractions in Section 2.1 one can easily obtain examples of nonuniform exponential dichotomies.

Example 3.3. For $\lambda<a<0$, the linear equation in $\mathbb{R}^{2}$ given by

$$
u^{\prime}=(\lambda+a t \sin t) u, \quad v^{\prime}=(-\lambda-a t \sin t) v
$$

admits a nonuniform exponential dichotomy. This can be shown by repeating arguments in Example 2.3. Furthermore, equation (26) does not admit a (uniform) exponential dichotomy. First observe that

$$
u(t)=e^{(\lambda-a)(t-s)-a t(\cos t-1)+a s(\cos s-1)-a(\sin s-\sin t)} u(s) .
$$

Given integers $m>n$, for $t=2 m \pi$ and $s=(2 n+1) \pi$ we have

$$
u(t)=e^{(\lambda-a)(t-s)-a s} u(s) .
$$

This shows that the first equation in (26) does not admit a uniform exponential contraction. Analogous arguments apply to the second equation.

When equation (7) admits a nonuniform exponential dichotomy, we consider the linear subspaces

$$
E(t)=P(t) X \quad \text { and } \quad F(t)=Q(t) X
$$

for each $t \geq 0$. These are called stable and unstable subspaces at $t$ (although strictly speaking $F(t)$ should be called unstable space only when $\underline{b}>0$ ). Clearly,

$$
X=E(t) \oplus F(t)
$$

for each $t \geq 0$, and the dimensions $\operatorname{dim} E(t)$ and $\operatorname{dim} F(t)$ are independent of $t$. Set

$$
\alpha(t)=\inf \{\|x-y\|: x \in E(t), y \in F(t),\|x\|=\|y\|=1\}
$$


(we took this notion from [39]). One can easily verify that in the case of Hilbert spaces we have

$$
\alpha(t)=2 \sin (\theta(t) / 2),
$$

where $\theta(t)$ is the angle between the subspaces $E(t)$ and $F(t)$. Furthermore, one can show that

$$
\frac{1}{\|P(t)\|} \leq \alpha(t) \leq \frac{2}{\|P(t)\|} \quad \text { and } \quad \frac{1}{\|Q(t)\|} \leq \alpha(t) \leq \frac{2}{\|Q(t)\|}
$$

for every $t \geq 0$. Setting $t=s$ in (22) we obtain

$$
\|P(t)\| \leq D e^{\varepsilon t} \text { and }\|Q(t)\| \leq D e^{\varepsilon t} .
$$

Therefore, by (28),

$$
\alpha(t) \geq e^{-\varepsilon t} / D \text { for every } t \geq 0 .
$$

This shows, at least in the case of Hilbert spaces, that when there exists a nonuniform exponential dichotomy the angle between the stable and unstable subspaces cannot decrease more than exponentially, with maximal exponential rate given by the number $\varepsilon$ in (21). In the general case of Banach spaces, $\alpha(t)$ in $(27)$ can also be thought of as an "angle" between the spaces $E(t)$ and $F(t)$. We mote that in the particular case of uniform exponential dichotomies we can take $\varepsilon=0$ in (28), and thus also in (29). This means that in the uniform case the "angle" between the stable and unstable subspaces is uniformly bounded from below for all time.

\subsection{Robustness of nonuniform exponential dichotomies}

We consider continuous functions $A, B: \mathbb{R}_{0}^{+} \rightarrow B(X)$, and equation (6). Set $\lambda=\min \{-\bar{a}, \underline{b}\}$. For a given constant $\delta>0$ we define

$$
\tilde{\lambda}=\lambda \sqrt{1-2 \delta D / \lambda} \text { and } \quad \tilde{D}=\frac{4 D^{2}}{1-\delta D /(\tilde{\lambda}+\lambda)} .
$$

Then for every $t \geq s \geq 0$ we have

$$
\|T(t, s) P(s)\| \leq D e^{-\lambda(t-s)+\varepsilon s}, \quad\left\|T(t, s)^{-1} Q(t)\right\| \leq D e^{-\lambda(t-s)+\varepsilon t} .
$$

The following result establishes the robustness of nonuniform exponential dichotomies for sufficiently small perturbations $B$.

Theorem 3.4 ([18, Theorem 2]). Assume that:

1. equation (7) admits a nonuniform exponential dichotomy with $\underline{b}>0$ and $\varepsilon<\lambda$;

2. $\|B(t)\| \leq \delta e^{-2 \varepsilon t}$ for every $t \geq 0$. 
For each sufficiently small $\delta$, equation (6) admits a nonuniform exponential dichotomy with the constants $D, \lambda$, and $\varepsilon$ in (31) replaced respectively by $\tilde{D}, \tilde{\lambda}$, and $2 \varepsilon$.

We note that by (30) the constants $\tilde{D}$ and $\tilde{\lambda}$ vary continuously with $\delta$.

Some arguments in the proof of Theorem 3.4 are inspired in work of Popescu in [63] in the case of uniform exponential dichotomies. Incidentally, we note that he uses the notion usually called admissibility, while we do not need it, of course independently of its possible interest in other situations. This notion goes back to Perron and refers to the characterization of the existence of an exponential dichotomy in terms of the existence and uniqueness of bounded solutions of the equation

$$
v^{\prime}=A(t) v+g(t)
$$

for $g$ in a certain class of bounded perturbations.

In the case of uniform exponential dichotomies the study of the robustness problem has a long history, in particular with work of Massera and Schäffer [49] (building on earlier work of Perron [57]; see also [50]), Coppel [26], and Dalec'kiľ and Kreı̆ [28] (in the case of Banach spaces), with different approaches and successive generalizations. For more recent work we refer to $[22,53,63,62]$ and the references therein. In particular, Chow and Leiva [22] and Pliss and Sell [62] considered the context of linear skewproduct flows and gave examples of applications in infinite dimension, including to parabolic partial differential equations and functional differential equations. We emphasize that in all these works it is only considered the case of uniform exponential dichotomies.

\section{Stable invariant manifolds}

This section is dedicated to the construction of stable invariant manifolds for sufficiently small perturbations of linear differential equations admitting a nonuniform exponential dichotomy. In other words, we describe versions of the stability results in Section 2.2 when not all directions are contracting. The existence of stable invariant manifolds corresponds precisely to the existence of an asymptotic stability along a certain invariant manifold, that is tangent to the stable space.

\subsection{Lipschitz stable invariant manifolds}

Before discussing the existence of smooth stable manifolds, we consider the simpler problem of existence of Lipschitz stable invariant "manifolds", i.e., graphs of Lipschitz functions along which we have asymptotic stability. 
Let again $X$ be a Banach space, and let $A: \mathbb{R}_{0}^{+} \rightarrow B(X)$ and $f: \mathbb{R}_{0}^{+} \times$ $X \rightarrow X$ be continuous functions such that (15) and (16) hold. In particular, $v(t)=0$ is still a solution of the equation $v^{\prime}=A(t) v+f(t, v)$.

One can easily verify that when $f$ is differentiable condition (16) is equivalent to the existence of constants $c>0$ and $q>0$ such that

$$
\left\|\frac{\partial f}{\partial v}(t, v)\right\| \leq c\|v\|^{q} \text { for every } t \geq 0 \text { and } v \in X .
$$

Condition (16) may sometimes be obtained with an appropriate cut-off of the perturbation in a neighborhood of $0 \in X$ and taking a Taylor expansion, provided that the perturbation is sufficiently regular. We note that since all norms in $\mathbb{R}^{2}$ are equivalent, when $q>1$ the $q$-norm $\left(\|u\|^{q}+\|v\|^{q}\right)^{1 / q}$ is equivalent to the 1-norm $\|u\|+\|v\|$. In this case, one can replace the factor $\|u\|^{q}+\|v\|^{q}$ in (16) by $(\|u\|+\|v\|)^{q}$, up to a multiplicative constant.

Given $s \geq 0$ and $v_{s} \in X$ we denote by

$$
(x(t), y(t))=\left(x\left(t, s, v_{s}\right), y\left(t, s, v_{s}\right)\right) \in E(t) \times F(t)
$$

the unique solution of the initial value problem in (17), i.e., of the problem

$$
\begin{aligned}
& x(t)=T(t, s) P(s) v_{s}+\int_{s}^{t} T(t, r) P(r) f(r, x(r), y(r)) d r, \\
& y(t)=T(t, s) Q(s) v_{s}+\int_{s}^{t} T(t, r) Q(r) f(r, x(r), y(r)) d r
\end{aligned}
$$

for $t \geq s$. The flow generated by the autonomous equation

$$
t^{\prime}=1, \quad v^{\prime}=A(t) v+f(t, v)
$$

is given by

$$
\Psi_{\tau}\left(s, v_{s}\right)=\left(s+\tau, x\left(s+\tau, s, v_{s}\right), y\left(s+\tau, s, v_{s}\right)\right), \quad \tau \geq 0 .
$$

The stable manifolds are obtained as graphs of Lipschitz functions. We first describe an appropriate class of functions. Let

$$
\alpha=\varepsilon(1+2 / q),
$$

with $\varepsilon$ as in (21). We fix $\delta>0$ and we consider the set of initial conditions

$$
X_{\alpha}=\left\{(s, \xi): s \geq 0 \text { and } \xi \in B_{s}\left(\delta e^{-\alpha s}\right)\right\} \text {, }
$$

where $B_{s}(\delta) \subset E(s)$ is the open ball of radius $\delta>0$ centered at zero. We denote by $X_{\alpha}$ the space of continuous functions $\varphi: X_{\alpha} \rightarrow X$ such that for each $s \geq 0$ and $\xi, \bar{\xi} \in B_{s}\left(\delta e^{-\alpha s}\right)$ we have

$$
\varphi(s, 0)=0, \quad \varphi\left(s, B_{s}\left(\delta e^{-\alpha s}\right)\right) \subset F(s),
$$

and

$$
\|\varphi(s, \xi)-\varphi(s, \bar{\xi})\| \leq\|\xi-\bar{\xi}\| .
$$


Given a function $\varphi \in \mathcal{X}_{\alpha}$ we consider its graph

$$
\mathcal{V}=\left\{(s, \xi, \varphi(s, \xi)):(s, \xi) \in X_{\alpha}\right\} \subset \mathbb{R}_{0}^{+} \times X .
$$

We note that $(s, 0) \in \mathcal{V}($ since $\varphi(s, 0)=0)$, for every $s \geq 0$. In particular, $\mathcal{V}$ contains the line $\mathbb{R}_{0}^{+} \times\{0\}$ and the graph

$$
\mathcal{V}_{s}=\left\{(s, \xi, \varphi(s, \xi)): \xi \in B_{s}\left(\delta e^{-\alpha s}\right)\right\}
$$

for each $s \geq 0$ (see Figure 1). We also set

$$
\beta=\alpha+\varepsilon=\varepsilon(2+2 / q),
$$

and we consider the corresponding sets $X_{\beta}$ and $x_{\beta}$, obtained replacing $\alpha$ by $\beta$ in the definitions. We will see that there exists a (unique) function $\varphi \in X_{\alpha}$ such that for every $(s, \xi) \in X_{\beta} \subset X_{\alpha}$ the solution of (17) with $v_{s}=(\xi, \varphi(s, \xi))$ is contained in $\mathcal{V}$. This means that for this function $\varphi$ the set $\mathcal{V}$ is forward invariant under the flow $\Psi_{\tau}$ in (33).

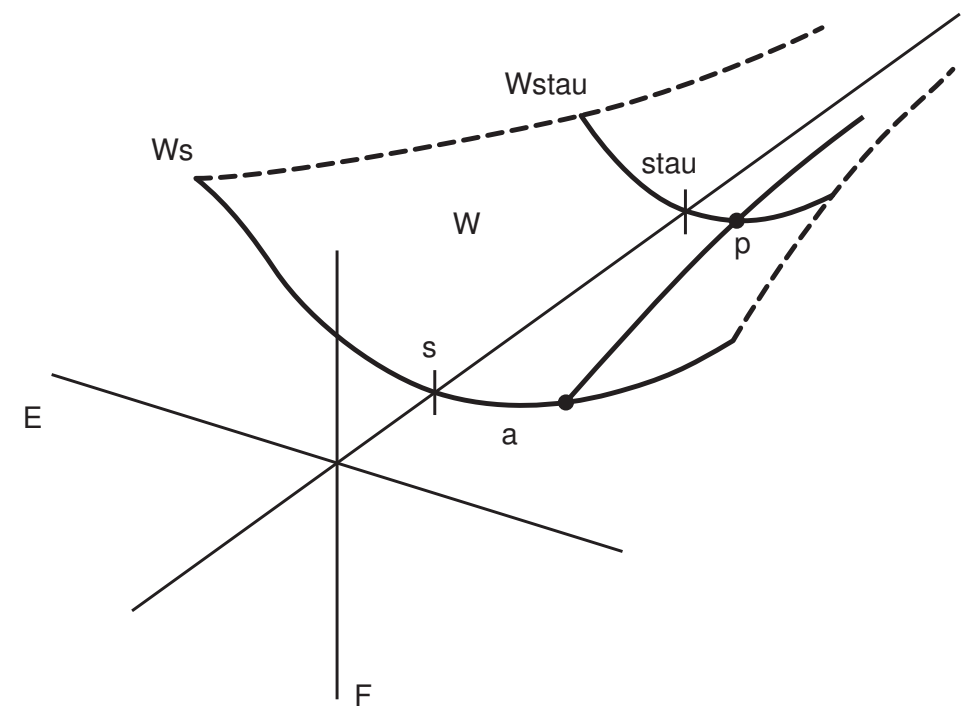

Figure 1. A stable manifold $V$ of the origin. In order that $\mathcal{V}$ is invariant under the flow $\Psi_{\tau}$ we require that $p=\Psi_{\tau}(s, \xi, \varphi(s, \xi))$. Here the subspaces $E=E(t)$ and $F=F(t)$ are assumed to be independent of $t$.

The following result establishes the existence of Lipschitz stable manifolds. 
Theorem 4.1 ([10, Theorem 3]). If equation (7) admits a nonuniform exponential dichotomy with

$$
\bar{a}+\alpha<0 \text { and } \bar{a}+\varepsilon<\underline{b} \text {, }
$$

then there exist $\delta>0$ and a unique function $\varphi \in \mathcal{X}_{\alpha}$ such that

$$
\Psi_{\tau}(s, \xi, \varphi(s, \xi)) \in \mathcal{V} \text { for every }(s, \xi) \in X_{\beta} \text { and } \tau \geq 0 .
$$

Furthermore, there exists $K>0$ such that for every $s \geq 0, \xi, \bar{\xi} \in B_{s}\left(\delta e^{-\beta s}\right)$, and $\tau \geq 0$ we have

$$
\left\|\Psi_{\tau}(s, \xi, \varphi(s, \xi))-\Psi_{\tau}(s, \bar{\xi}, \varphi(s, \bar{\xi}))\right\| \leq K e^{\bar{a} \tau+\varepsilon s}\|\xi-\bar{\xi}\| .
$$

We call to the set $\mathcal{V}$ in (36) a Lipschitz stable manifold of the origin for equation (17). In particular, setting $\bar{\xi}=0$ in (39) we find that any solution of the initial value problem in (17) starting in $\mathcal{V}$, i.e., with $v_{s}=(\xi, \varphi(s, \xi))$ for some $\xi \in B_{s}\left(\delta e^{-\beta s}\right)$, approaches zero with exponential speed $\bar{a}$ (which is independent of $s$ and $\xi$ ). We note that both inequalities in (38) are automatically satisfied when $\varepsilon$ is sufficiently small, and that the first one is satisfied for a given $\varepsilon<|\bar{a}|$ provided that $q$ is sufficiently large (i.e., provided that the order of the perturbation is sufficiently large).

We now briefly explain how Theorem 4.1 can be used to establish the existence of stable invariant manifolds for nonuniformly hyperbolic trajectories. Consider a $C^{1}$ function $F: \mathbb{R}_{0}^{+} \times X \rightarrow X$ and the equation

$$
v^{\prime}=F(t, v) \text {. }
$$

We say that a solution $v_{0}(t)$ of (40) is nonuniformly hyperbolic if the linear equation in (7) with

$$
A(t)=\frac{\partial F}{\partial v}\left(t, v_{0}(t)\right)
$$

admits a nonuniform exponential dichotomy. We continue to assume that all solutions of equation (7) are global. The following statement is a simple consequence of Theorem 4.1.

Theorem 4.2. Let $v_{0}(t)$ be a nonuniformly hyperbolic solution of equation (40). If there exist constants $c>0$ and $q>0$ such that

$$
\left\|\frac{\partial F}{\partial v}\left(t, y+v_{0}(t)\right)-A(t)\right\| \leq c\|y\|^{q}
$$

for every $t \geq 0$ and $y \in X$, and (38) holds, then there exist $\delta>0$ and $a$ unique function $\varphi \in X_{\beta}$ such that the set

$$
\mathcal{W}=\left\{(s, \xi, \varphi(s, \xi))+\left(0, v_{0}(s)\right):(s, \xi) \in X_{\alpha}\right\}
$$

has the following properties: 
1. if

$$
\left(s, v_{s}\right) \in\left\{(s, \xi, \varphi(s, \xi))+\left(0, v_{0}(s)\right):(s, \xi) \in X_{\beta}\right\},
$$

then $(t, v(t)) \in \mathcal{W}$ for every $t \geq s$, where $v(t)=v\left(t, v_{s}\right)$ is the unique solution of (40) with $v(s)=v_{s}$;

2. there exists $K>0$ such that for every $s \geq 0,\left(s, v_{s}\right),\left(s, \bar{v}_{s}\right) \in \mathcal{W}$, and $t \geq s$ we have

$$
\left\|v\left(t, v_{s}\right)-v\left(t, \bar{v}_{s}\right)\right\| \leq K e^{\bar{a}(t-s)+\varepsilon s}\left\|v_{s}-\bar{v}_{s}\right\| .
$$

We call to the set $\mathcal{W}$ in (41) a Lipschitz stable manifold of $v_{0}(t)$.

\subsection{Smooth stable manifolds}

We now discuss the existence of smooth stable invariant manifolds. Theorem 4.1 already shows that the function $\varphi$ in (36) is unique in a class of Lipschitz functions. Thus, to show that the set $\mathcal{V}$ in (36) is a smooth manifold it is sufficient to show that $\varphi$ is smooth. However, it is more convenient to deal with the problem from the beginning, finding independently a unique function $\varphi$, now in an appropriate class of smooth functions, that a posteriori must coincide with the function $\varphi$ already obtained in Theorem 4.1. The main novelty in our approach, besides considering the nonuniform setting, is the method of proof, which uses a single fixed point problem to obtain the maximal degree of regularity for the function $\varphi$. We refer to Section 4.3 for details on the method of proof.

We assume in this section that $A: \mathbb{R}_{0}^{+} \rightarrow B(X)$ and $f: \mathbb{R}_{0}^{+} \times X \rightarrow X$ are of class $C^{k}$. We continue to denote by $B_{s}(\delta) \subset E(s)$ be the open ball of radius $\delta>0$ centered at zero. Given $q>k$ we set

$$
\alpha=\frac{\varepsilon(q+k+2)}{q-k}>\varepsilon \quad \text { and } \quad \beta=\frac{\varepsilon(2 q+2)}{q-k}>\alpha .
$$

We notice that when $k=0$ the numbers $\alpha$ and $\beta$ coincide respectively with $\alpha$ and $\beta$ in (34) and (37). We continue to consider the set $X_{\alpha}$ in (35).

We assume that there exist constants $c, \delta^{\prime}>0, q>k$, and $\eta \in(0,1]$ such that for every $t \geq 0$ and $u, v \in X$ we have:

1.

$$
f(t, 0)=0, \quad \frac{\partial f}{\partial v}(t, 0)=0, \quad f \mid Y_{\beta}=0
$$

where

$$
Y_{\beta}=\left\{(s, v) \in \mathbb{R}_{0}^{+} \times X:\|v\| \geq \delta^{\prime} e^{-\beta s}\right\}
$$

2.

$$
\left\|\frac{\partial^{j} f}{\partial v^{j}}(t, u)\right\| \leq c\|u\|^{q+1-j} \text { for } j=1, \ldots, k
$$


and

$$
\left\|\frac{\partial^{k} f}{\partial v^{k}}(t, u)-\frac{\partial^{k} f}{\partial v^{k}}(t, v)\right\| \leq c\|u-v\|^{\eta}(\|u\|+\|v\|)^{q-k} .
$$

The last condition in (42) may sometimes be obtained with an appropriate cut-off of the function $f$ (see also the discussion after Theorem 4.3).

We denote by $z_{\alpha}$ the space of continuous functions $\varphi: X_{\alpha} \rightarrow X$ of class $C^{k}$ in $\xi$ such that for each $s \geq 0$ and $\xi, \bar{\xi} \in B_{s}\left(\delta e^{-\alpha s}\right)$ we have:

1.

$$
\varphi(s, 0)=0, \quad \frac{\partial \varphi}{\partial \xi}(s, 0)=0, \quad \varphi\left(s, B_{s}\left(\delta e^{-\alpha s}\right)\right) \subset F(s)
$$

2.

$$
\left\|\frac{\partial^{j} \varphi}{\partial \xi^{j}}(s, \xi)\right\| \leq 1 \text { for } j=1, \ldots, k
$$

and

$$
\left\|\frac{\partial^{k} \varphi}{\partial \xi^{k}}(s, \xi)-\frac{\partial^{k} \varphi}{\partial \xi^{k}}(s, \bar{\xi})\right\| \leq\|\xi-\bar{\xi}\|^{\eta} .
$$

For each function $\varphi \in z_{\alpha}$ we consider its graph $\mathcal{V}$ given by (36).

We now formulate a stable manifold theorem. Set $p_{s, \xi}=(s, \xi, \varphi(s, \xi))$.

Theorem 4.3 ([9, Theorem 1]). If equation (7) admits a nonuniform exponential dichotomy with

$$
\bar{a}+\alpha<0 \text { and } \bar{a}+\varepsilon<\underline{b},
$$

then there exist $\delta, \delta^{\prime}>0$ and a unique function $\varphi \in z_{\alpha}$ such that

$$
\Psi_{\tau}\left(p_{s, \xi}\right) \in \mathcal{V} \text { for every }(s, \xi) \in X_{\beta} \text { and } \tau \geq 0 .
$$

\section{Furthermore:}

1. $\mathcal{V}$ is a manifold of class $C^{k}$ that contains the line $\mathbb{R}_{0}^{+} \times\{0\}$ and satisfies $T_{(s, 0)} \mathcal{V}=\mathbb{R} \times E(s)$ for every $s \geq 0$;

2. there exists $K>0$ such that for every $s \geq 0, \xi, \bar{\xi} \in B_{s}\left(\delta e^{-\beta s}\right)$, and $\tau \geq 0$ we have

$$
\left\|\frac{\partial^{j} \Psi_{\tau}}{\partial \xi^{j}}\left(p_{s, \xi}\right)-\frac{\partial^{j} \Psi_{\tau}}{\partial \xi^{j}}\left(p_{s, \bar{\xi}}\right)\right\| \leq K e^{\bar{a} \tau+\varepsilon(j+1) s}\|\xi-\bar{\xi}\|
$$

for $j=0, \ldots, k-1$, and

$$
\left\|\frac{\partial^{k} \Psi_{\tau}}{\partial \xi^{k}}\left(p_{s, \xi}\right)-\frac{\partial^{k} \Psi_{\tau}}{\partial \xi^{k}}\left(p_{s, \bar{\xi}}\right)\right\| \leq K e^{\bar{a} \tau+\varepsilon(k+1) s}\|\xi-\bar{\xi}\|^{\eta} .
$$


We observe that both inequalities in (43) hold when $\varepsilon$ is sufficiently small. Note that we also establish the exponential decay on the stable manifold of the derivatives up to order $k-1$ of the flow with respect to the initial condition (see (44)). We are not aware of any similar result in the literature, even in the case of uniform exponential dichotomies. The exponential decay of the derivatives can be understood in the following manner. When we consider higher-order jets (other than the first, which corresponds to the linear variational equation), the higher-order linearizations maintain the linear part $A(t)$ of the linear variational equation. Thus, the higher-order jets possess essentially the same nonuniform exponential dichotomies, although in higher-dimensional spaces. This explains the exponential behavior of the derivatives along the stable manifolds.

We now discuss an alternative version of the stable manifold theorem, partly motivated by the desire of eliminating the third condition in (42). In fact this condition requires an exponential decay on time for the function $f$ and its derivatives. We consider a function $f: \mathbb{R}_{0}^{+} \times X \rightarrow X$ of class $C^{k}$ such that:

1. $f(t, 0)=0$ and $\partial f / \partial v(t, 0)=0$ for every $t \geq 0$;

2. for some constant $\delta>0$, and every $t \geq 0$ and $u, v \in X$ we have

$$
\left\|\frac{\partial^{j} f}{\partial v^{j}}(t, u)\right\| \leq \delta e^{-(k+2) \varepsilon t} \text { for } j=0, \ldots, k-1,
$$

and

$$
\left\|\frac{\partial^{k} f}{\partial v^{k}}(t, u)-\frac{\partial^{k} f}{\partial v^{k}}(t, v)\right\| \leq \delta e^{-(k+2) \varepsilon t}\|u-v\| .
$$

Let also $X$ be the space of continuous functions

$$
\varphi:\left\{(s, \xi) \in \mathbb{R}_{0}^{+} \times X: \xi \in E(s)\right\} \rightarrow X
$$

of class $C^{k}$ in $\xi$ such that for each $s \geq 0$ and $\xi, \bar{\xi} \in E(s)$ we have

$$
\varphi(s, 0)=0, \quad \frac{\partial \varphi}{\partial \xi}(s, 0)=0, \quad \varphi(s, E(s)) \subset F(s),
$$

and

$$
\left\|\frac{\partial^{j} \varphi}{\partial \xi^{j}}(s, \xi)-\frac{\partial^{j} \varphi}{\partial \xi^{j}}(s, \bar{\xi})\right\| \leq\|\xi-\bar{\xi}\| \text { for } j=0, \ldots, k-1 .
$$

For each $\varphi \in \mathcal{X}$ we consider its graph

$$
\mathcal{W}=\left\{(s, \xi, \varphi(s, \xi)):(s, \xi) \in \mathbb{R}_{0}^{+} \times E(s)\right\} \subset \mathbb{R}_{0}^{+} \times X .
$$

We now formulate a second stable manifold theorem. We continue to write $p_{s, \xi}=(s, \xi, \varphi(s, \xi))$. 
Theorem 4.4. If equation (7) admits a nonuniform exponential dichotomy and $\bar{a}+\varepsilon<\underline{b}$, then there exist $\delta>0$ and a unique function $\varphi \in X$ such that $\Psi_{\tau}(\mathcal{W}) \subset \mathcal{W}$ for every $\tau \geq 0$. Furthermore:

1. $\mathcal{W}$ is a $C^{k}$ manifold with $T_{(s, 0)} \mathcal{W}=\mathbb{R} \times E(s)$ for every $s \geq 0$;

2. there exists $K>0$ such that for every $s \geq 0, \xi, \bar{\xi} \in E(s), \tau \geq 0$, and $j=0, \ldots, k$ we have

$$
\left\|\frac{\partial^{j} \Psi_{\tau}}{\partial \xi^{j}}\left(p_{s, \xi}\right)-\frac{\partial^{j} \Psi_{\tau}}{\partial \xi^{j}}\left(p_{s, \bar{\xi}}\right)\right\| \leq K e^{\bar{a} \tau+(j+1) \varepsilon s}\|\xi-\bar{\xi}\| .
$$

For $k=1$, this statement is Theorem 1 in [17]. The proof for an arbitrary $k$ follows essentially the same steps as in the proof of Theorem 4.3 (see Section 4.3 for details).

When equation (7) admits a strong nonuniform exponential dichotomy (see Definition 3.2) we also obtain a characterization of the stable manifold in terms of the exponential growth rate of solutions.

Theorem 4.5 ([17, Theorem 2]). Assume that equation (7) admits a strong nonuniform exponential dichotomy with $\underline{b}>0$. Given $s \geq 0$ and $v \in X$, if

$$
\limsup _{t \rightarrow+\infty} \frac{1}{t} \log \left\|\Psi_{t}(s, v)\right\| \leq \underline{b}-2 \varepsilon
$$

then $(s, v) \in \mathcal{W}$.

\subsection{Method of proof}

Our approach to prove the stable manifold theorems (Theorems 4.3 and 4.4) starts by using the differential equation and the desired invariance of the manifold to conclude that it must be the graph of a function satisfying a certain fixed point problem (as in many other stable manifold theorems). However, the extra small exponentials of a nonuniform exponential dichotomy led us to consider two fixed-point problems - one to obtain an a priori bound for the stable component of the solutions along each graph, and the other to obtain the graph that gives the stable manifold. To obtain the estimates in the fixed point problems, we need sharp bounds for several derivatives, such as for example for the derivatives of the vector field along each graph, i.e., of the vector field

$$
(t, \xi) \mapsto f(t, \xi, \varphi(t, \xi)) .
$$

For this we use a multivariate version of the Faà di Bruno formula in [25] for the derivatives of a composition. Consider open sets $Y, Z$, and $W$ of Banach spaces. Let $g: Y \rightarrow Z$ be defined in a neighborhood of $x \in Y$ with derivatives up to order $n$ at $x$. Let also $f: Z \rightarrow W$ be defined in a 
neighborhood of $y=g(x) \in Z$ with derivatives up to order $n$ at $y$. The $n$-th derivative of the composition $h=f \circ g$ at $x$ is given by

$$
d_{x}^{n} h=\sum_{k=1}^{n} d_{y}^{k} f \sum_{\substack{0 \leq r_{1}, \ldots, r_{k} \leq n \\ r_{1}+\cdots+r_{k}=n}} c_{r_{1} \cdots r_{k}} d_{x}^{r_{1}} g \cdots d_{x}^{r_{k}} g
$$

for some nonnegative integers $c_{r_{1} \cdots r_{k}}$. The formula allows us to estimate the norm of the derivatives of a composition in terms of the original functions. Namely, collecting derivatives of equal order, one can show that for each $n \in \mathbb{N}$ there exists $c=c(n)>0$ such that (see [25])

$$
\left\|d_{x}^{n} h\right\| \leq c \sum_{k=1}^{n}\left\|d_{y}^{k} f\right\| \sum_{p(n, k)} \prod_{j=1}^{n}\left\|d_{x}^{j} g\right\|^{k_{j}}
$$

where

$$
p(n, k)=\left\{\left(k_{1}, \ldots, k_{n}\right) \in \mathbb{N}_{0}^{n}: \sum_{j=1}^{n} k_{j}=k \text { and } \sum_{j=1}^{n} j k_{j}=n\right\}
$$

(here $\mathbb{N}_{0}$ is the set of nonnegative integers). A multivariate extension of the Faà di Bruno formula in (45) was established in [25]. Although several special cases were treated before, the general formula for the derivatives of a composition was first obtained by Faà di Bruno in [30]. We recommend [46] for the history of the problem and for many related references.

In the proof of the stable manifold theorems we also use a result of Elbialy in [29] (see Theorem 4.6), that goes back to a lemma of Henry in [38], and which allows us to establish simultaneously the existence and regularity of the stable manifolds using a single fixed point problem, instead of one for each of the successive derivatives. We now briefly describe Henry's lemma. Let $X$ and $Y$ be Banach spaces, and let $U \subset X$ be an open set. Given constants $\eta \in(0,1], k \in \mathbb{N} \cup\{0\}$, and $c>0$ we define the set

$$
C_{c}^{k, \eta}(U, Y)=\left\{u \in C^{k, \eta}(U, Y):\|u\|_{k, \eta} \leq c\right\},
$$

where $C^{k, \eta}(U, Y)$ is the space of $C^{k}$ functions $u: U \rightarrow Y$ with $\eta$-Hölder continuous $k$-th derivative, and with the norm

$$
\|u\|_{k, \eta}=\max \left\{\|u\|_{\infty},\|d u\|_{\infty}, \ldots,\left\|d^{k} u\right\|_{\infty}, H_{\eta}\left(d^{k} u\right)\right\},
$$

where $\|\cdot\|_{\infty}$ denotes the supremum norm and

$$
H_{\eta}(u)=\sup \left\{\frac{\|u(x)-u(y)\|}{\|x-y\|^{\eta}}: x, y \in X \text { and } x \neq y\right\} .
$$


The following result shows that $C_{c}^{k, \eta}(U, Y)$ is closed in the space of continuous functions $C(U, Y)$ with the supremum norm.

Theorem 4.6 (see $[38,29]$ ). Let $X$ and $Y$ be Banach spaces, and let $U \subset X$ be an open set. If $u_{n} \in C_{c}^{k, \eta}(U, Y)$ for each $n \in \mathbb{N}$ and $u: U \rightarrow Y$ is a function such that $\left\|u_{n}-u\right\|_{\infty} \rightarrow 0$ as $n \rightarrow \infty$, then $u \in C_{c}^{k, \eta}(U, Y)$ and for each $x \in U$ we have $d^{k} u_{n}(x) \rightarrow d^{k} u(x)$ as $n \rightarrow \infty$.

When $k=1$ the statement in Theorem 4.6 was first established by Henry in [38, Lemma 6.1.6]. A related result was obtained by Lanford in [44, Lemma 2.5]. In particular, Theorem 4.6 says that the closed unit ball in $C^{k, \eta}$ is closed with respect to the $C^{0}$-topology. This property allows us to consider contraction maps solely using the supremum norm, instead of any norm that also involves the derivatives. For example, it follows in a straightforward manner from Theorem 4.6 that the space $Z_{\alpha}$ (see Section 4.2) with the norm

$$
\|\varphi\|=\sup \left\{\frac{\|\varphi(s, \xi)\|}{\|\xi\|}: s \geq 0 \text { and } \xi \in B_{s}\left(\delta e^{-\alpha s}\right) \backslash\{0\}\right\}
$$

is a complete metric space. The function $\varphi \in Z_{\alpha}$ in Theorem 4.3 is precisely obtained as the unique fixed point of a map in $z_{\alpha}$ that is a contraction with the norm in (46).

In $[5,4]$ we presented an alternative proof of the stable manifold theorem with slightly weaker assumptions, although only in the finite-dimensional case. This is due to the fact that we use in a decisive manner the compactness of the closed unit ball in $\mathbb{R}^{n}$. The proof is based on the construction of an invariant family of cones along each orbit, as in the uniform hyperbolic theory, although now using a family of Lyapunov norms. The family of cones allows us to obtain an invariant distribution that coincides with the tangent space of the stable manifold. This procedure also allows us to discuss the continuity of the distribution, and thus the continuity of the tangent spaces, which corresponds to the $C^{1}$ regularity of the stable manifold.

\section{Construction of conjugacies}

We address in this section the construction of topological conjugacies between the flows defined by the equations

$$
v^{\prime}=A(t) v \quad \text { and } \quad v^{\prime}=A(t) v+f(t, v),
$$

in the general case when the linear equation admits a nonuniform exponential dichotomy. We also show that it is always possible to construct Hölder 
continuous conjugacies. We refer to [15] for the construction of topological (and Hölder continuous) conjugacies between nonautonomous dynamics with distinct linear parts.

\subsection{Conjugacies for flows}

Let $X$ be a Banach space. We continue to denote by $B(X)$ the set of bounded linear operators in $X$, and we consider a continuous function $A: \mathbb{R} \rightarrow B(X)$ such that each solution of (7) is defined for every $t \in \mathbb{R}$. We also consider a continuous function $f: \mathbb{R} \times X \rightarrow X$ and we assume that there exists $\delta>0$ such that for every $t \in \mathbb{R}$ and $x, y \in X$ we have

$$
\|f(t, x)-f(t, y)\| \leq \delta e^{-6 \varepsilon|t|} \min \{1,\|x-y\|\}
$$

(with the constant $\varepsilon$ as in (23)). The equations in (47) define evolution operators that we denote respectively by $T(t, s)$ and $R(t, s)$, for $t, s \in \mathbb{R}$.

Theorem 5.1 ([15, Theorem 7$]$ ). If equation (7) admits a strong nonuniform exponential dichotomy in $\mathbb{R}$ with $\underline{b}>0$, and $\delta$ is sufficiently small, then there exist homeomorphisms $h_{t}: X \rightarrow X$ for $t \in \mathbb{R}$ such that

$$
T(t, s) \circ h_{s}=h_{t} \circ R(t, s), \quad t, s \in \mathbb{R} .
$$

It is also shown in [15] that the maps $h_{t}$ are unique among those that are close to the identity for each $t$. More precisely, the maps $h_{t}-$ Id are uniformly bounded in $t$ with respect to a family of Lyapunov norms. We refer to Section 5.2 for a detailed discussion in the case of discrete time.

The original references for the Grobman-Hartman theorem (in the case of uniformly hyperbolic dynamics) are Grobman [32, 33] and Hartman [36, 37]. Using the ideas in Moser's proof in [52] of the structural stability of Anosov diffeomorphisms, the Grobman-Hartman theorem was extended to Banach spaces independently by Palis [55] and Pugh [64]. In the case of continuous time a version of the Grobman-Hartman theorem for nonautonomous differential equations $v^{\prime}=A(t) v$ was obtained by Palmer in [56] (with the exception of the Hölder property of the conjugacies), although assuming the existence of a uniform exponential dichotomy.

We can also show that the topological conjugacies $h_{t}$ and their inverses are Hölder continuous. We assume that equation (7) admits a strong nonuniform exponential dichotomy and we set

$$
\alpha_{0}=\min \{\bar{a} / \underline{a}, \underline{b} / \bar{b}\} .
$$

It follows from $(23)$ that $\alpha_{0} \in(0,1]$.

Theorem 5.2 ([15, Theorem 7$]$ ). If equation (7) admits a strong nonuniform exponential dichotomy in $\mathbb{R}$ with $\underline{b}>0$, then for each $\alpha \in\left(0, \alpha_{0}\right)$ and 
each $\delta$ sufficiently small (depending on $\alpha$ ), there exist homeomorphisms $h_{t}$ as in Theorem 5.1, and a constant $K>0$ (depending on $\alpha$ and $\delta$ ) such that

$$
\left\|h_{t}(x)-h_{t}(y)\right\| \leq K e^{2 \varepsilon(2+3 \alpha)|t|}\|x-y\|^{\alpha}
$$

and

$$
\left\|h_{t}^{-1}(x)-h_{t}^{-1}(y)\right\| \leq K e^{2 \varepsilon(2+3 \alpha)|t|}\|x-y\|^{\alpha}
$$

for every $t \in \mathbb{R}$ and $x, y \in X$ with $\|x-y\| \leq e^{-3 \varepsilon|t|}$.

We note that in the case of uniform exponential dichotomies, the Hölder regularity of the conjugacies seems to have been known by the experts for some time, although the first proof only appeared recently in [11]. The Hölder property was claimed by van Strien in [79, Proposition 4.6], but it was observed in [67] (see also [34]) that some problems in the proof are not yet overcome. This should be compared with the discussion in [34], where the authors indicate that the statement of the Hölder regularity is contained in a preprint of Belitskil [19] (we believe that the preprint circulates since 1994 but it remains unpublished).

\subsection{Conjugacies for maps}

The conjugacies for flows are constructed by first considering the time1 maps. After obtaining conjugacies for these maps, we use a general "integrating" procedure to obtain the desired conjugacies for the original flows.

We first consider arbitrary maps (not necessarily obtained from a flow). For each $m \in \mathbb{Z}$ consider:

1. an invertible linear operator $A_{m} \in B(X)$ with inverse $A_{m}^{-1} \in B(X)$;

2. a continuous map $f_{m}: X \rightarrow X$.

We now introduce the notion of nonuniform exponential dichotomy in the case of discrete time. Set

$$
\mathcal{A}(m, n)=A_{m-1} \cdots A_{n}, \quad m>n .
$$

Definition 5.3. We say that the sequence of linear operators $\left(A_{m}\right)_{m \in \mathbb{Z}}$ admits a nonuniform exponential dichotomy if there exist projections $P_{n} \in$ $B(X)$ for $n \in \mathbb{Z}$ with

$$
P_{m} \mathcal{A}(m, n)=\mathcal{A}(m, n) P_{n} \text { for every } m, n \in \mathbb{Z} \text { with } m \geq n,
$$

and there exist constants

$$
D>0, \quad \bar{a}<0 \leq \underline{b}, \quad \text { and } \quad \varepsilon \geq 0
$$

such that for every $m, n \in \mathbb{Z}$ with $m \geq n$ we have

$$
\left\|\mathcal{A}(m, n) P_{n}\right\| \leq D e^{\bar{a}(m-n)+\varepsilon|n|}, \quad\left\|\mathcal{A}(m, n)^{-1} Q_{m}\right\| \leq D e^{-\underline{b}(m-n)+\varepsilon|m|},
$$

where $Q_{n}=\mathrm{Id}-P_{n}$ are the complementary projections. 
For a sequence $\left(A_{m}\right)_{m \in \mathbb{Z}}$ admitting a nonuniform exponential dichotomy, we consider the linear subspaces

$$
E_{m}=P_{m} X \quad \text { and } \quad F_{m}=Q_{m} X
$$

for $m \in \mathbb{Z}$. Clearly, $X=E_{m} \oplus F_{m}$ for every $m \in \mathbb{Z}$, and the dimensions $\operatorname{dim} E_{m}$ and $\operatorname{dim} F_{m}$ are independent of $m$. We also define the operators

$$
B_{m}=A_{m} \mid E_{m}: E_{m} \rightarrow E_{m+1} \quad \text { and } \quad C_{m}=A_{m} \mid F_{m}: F_{m} \rightarrow F_{m+1}
$$

for each $m \in \mathbb{Z}$. Clearly, $B_{m}$ and $C_{m}$ are invertible continuous linear operators with continuous inverse. Furthermore, with respect to the decompositions $X=E_{m} \oplus F_{m}$ each operator $A_{m}$ has the form

$$
A_{m}=\left(\begin{array}{cc}
B_{m} & 0 \\
0 & C_{m}
\end{array}\right), \quad m \in \mathbb{Z}
$$

Setting, for each $m \geq n$,

$$
\mathcal{B}(m, n)=B_{m-1} \cdots B_{n} \quad \text { and } \quad \mathcal{C}(m, n)=C_{m-1} \cdots C_{n},
$$

we introduce Lyapunov norms as follows. Choose $\varrho>0$ such that $\varrho<$ $\min \{-\bar{a}, \underline{b}\}$. For each $m \in \mathbb{Z}$ we define the norms

$$
\|x\|_{m}^{\prime}=\sum_{k \geq m}\|\mathcal{B}(k, m) x\| e^{(-\bar{a}-\varrho)(k-m)} \quad \text { for } x \in E_{m}
$$

and

$$
\|y\|_{m}^{\prime}=\sum_{k \leq m}\left\|\mathcal{C}(m, k)^{-1} y\right\| e^{(\underline{b}-\varrho)(m-k)} \quad \text { for } y \in F_{m} .
$$

We also define

$$
\|(x, y)\|_{m}^{\prime}=\|x\|_{m}^{\prime}+\|y\|_{m}^{\prime} \text { for each }(x, y) \in E_{m} \times F_{m} .
$$

Finally, we consider the space $\mathcal{X}$ of sequences $u=\left(u_{m}\right)_{m \in \mathbb{Z}}$ of continuous functions $u_{m}: X \rightarrow X$ such that

$$
\sup \left\{\left\|u_{m}(x)\right\|_{m}^{\prime}: m \in \mathbb{Z}, x \in X\right\}<\infty .
$$

We finally assume that there exists $\delta>0$ such that for each $m \in \mathbb{Z}$ and $x, y \in X$ we have

$$
\left\|f_{m}(x)\right\| \leq \delta e^{-\varepsilon|m|}
$$

and

$$
\left\|f_{m}(x)-f_{m}(y)\right\| \leq \delta e^{-\varepsilon|m|}\|x-y\|
$$

(with the constant $\varepsilon$ as in (48)).

The following is a version of the Grobman-Hartman theorem in this nonuniformly hyperbolic setting. 
Theorem 5.4 ([8, Theorems 1 and 3 and Corollary 1]). If the sequence of linear operators $\left(A_{m}\right)_{m \in \mathbb{Z}}$ admits a nonuniform exponential dichotomy with $\underline{b}>0$, and $\delta$ is sufficiently small, then there exist unique sequences $\left(u_{m}\right)_{m \in \mathbb{Z}} \in \mathcal{X}$ and $\left(v_{m}\right)_{m \in \mathbb{Z}} \in \mathcal{X}$ such that for every $m \in \mathbb{Z}$ we have

$$
A_{m} \circ \widehat{u}_{m}=\widehat{u}_{m+1} \circ\left(A_{m}+f_{m}\right)
$$

and

$$
\widehat{v}_{m+1} \circ A_{m}=\left(A_{m}+f_{m}\right) \circ \widehat{v}_{m},
$$

where $\widehat{u}_{m}=\mathrm{Id}+u_{m}$ and $\widehat{v}_{m}=\mathrm{Id}+v_{m}$. Furthermore,

$$
\widehat{u}_{m} \circ \widehat{v}_{m}=\widehat{v}_{m} \circ \widehat{u}_{m}=\mathrm{Id}, \quad m \in \mathbb{Z} .
$$

The Hölder regularity of the conjugacies is also established in [8].

We now explain how we can obtain the conjugacies for the flows from the conjugacies for the time-1 maps. Fix $r \in[-1,1]$. For each $m \in \mathbb{Z}$ we consider the invertible linear operators

$$
A_{m}=T(m+r, m+r-1),
$$

and the continuous maps

$$
f_{m}(u)=\int_{m+r-1}^{m+r} T(m+r, \tau) f(\tau, v(\tau, u)) d \tau,
$$

where $v(t, u)$ is the solution of the differential equation $v^{\prime}=A(t) v+f(t, v)$ with $v(m+r-1)=u$. One can show in a simple manner that when the conditions in Theorem 5.1 hold we can apply Theorem 5.4 (in particular, the sequence of operators $\left(A_{m}\right)_{m \in \mathbb{Z}}$ admits a nonuniform exponential dichotomy). Therefore, there exist homeomorphisms $G_{m, r}: X \rightarrow X$ for $m \in \mathbb{Z}$ such that

$$
T(m+r, m+r-1) \circ G_{m, r}=G_{m+1, r} \circ R(m+r, m+r-1) .
$$

Furthermore, the uniqueness statement in Theorem 5.4 can be used to show that

$$
G_{m, r}=G_{\bar{m}, \bar{r}} \quad \text { whenever } \quad \bar{m}+\bar{r}=m+r .
$$

In other words, we can define homeomorphisms

$$
J_{t}=G_{m, r} \text { with } m=[t] \text { and } r=t-[t],
$$

where $[t]$ denotes the integer part of $t$. Finally, for each $t \in \mathbb{R}$ we define the $\operatorname{map} h_{t}: X \rightarrow X$ by

$$
h_{t}(x)=\int_{0}^{1}\left[T(t, r+t) \circ J_{\tau+t} \circ R(\tau+t, t)\right](x) d \tau .
$$

One can show that these maps are the required conjugacies, with the properties in Theorems 5.1 and 5.2 . 


\section{Center manifolds and reversibility}

This section is dedicated to the construction of invariant center manifolds. We also discuss their reversibility properties when the ambient dynamics is reversible.

\subsection{Existence of center manifolds}

Let $A: \mathbb{R} \rightarrow B(X)$ be a continuous function. We continue to assume that all solutions of equation (7) are global. We first introduce the notion of nonuniform exponential trichotomy.

Definition 6.1. We say that equation (7) admits a nonuniform exponential trichotomy if there exist functions $P, Q_{1}, Q_{2}: \mathbb{R} \rightarrow B(X)$ such that $P(t)$, $Q_{1}(t)$, and $Q_{2}(t)$ are projections with

$$
\begin{aligned}
P(t)+Q_{1}(t)+Q_{2}(t) & =\mathrm{Id}, \\
P(t) T(t, s)=T(t, s) P(s), \quad Q_{i}(t) T(t, s) & =T(t, s) Q_{i}(s), \quad i=1,2
\end{aligned}
$$

for every $t, s \in \mathbb{R}$, and there exist constants

$$
D, \varepsilon \geq 0, \quad 0 \leq a<b, \quad \text { and } \quad 0 \leq c<d
$$

such that:

1. for every $s, t \in \mathbb{R}$ with $t \geq s$ we have

$$
\|T(t, s) P(s)\| \leq D e^{a(t-s)+\varepsilon|s|}, \quad\left\|T(t, s)^{-1} Q_{2}(t)\right\| \leq D e^{-b(t-s)+\varepsilon|t|} ;
$$

2. for every $s, t \in \mathbb{R}$ with $t \leq s$ we have

$$
\|T(t, s) P(s)\| \leq D e^{c(s-t)+\varepsilon|s|}, \quad\left\|T(t, s)^{-1} Q_{1}(t)\right\| \leq D e^{-d(s-t)+\varepsilon|t|} .
$$

In a certain sense, the existence of a nonuniform exponential trichotomy is the weakest hypothesis under which one is able to establish the existence of invariant center manifolds.

We now present the hypotheses for the nonlinear part of the vector field. We assume that there is an integer $k \in \mathbb{N}$ such that $A: \mathbb{R} \rightarrow B(X)$ and $f: \mathbb{R} \times X \rightarrow X$ are of class $C^{k}$. We also assume that:

1. $f(t, 0)=0$ and $\partial f / \partial v(t, 0)=0$ for every $t \in \mathbb{R}$;

2. there exists $\delta>0$ such that for every $t \in \mathbb{R}$ and $u, v \in X$ we have

$$
\left\|\frac{\partial^{j} f}{\partial v^{j}}(t, u)\right\| \leq \delta e^{-(k+2) \varepsilon|t|} \text { for } j=1, \ldots, k,
$$

and

$$
\left\|\frac{\partial^{k} f}{\partial v^{k}}(t, u)-\frac{\partial^{k} f}{\partial v^{k}}(t, v)\right\| \leq \delta e^{-(k+2) \varepsilon|t|}\|u-v\| .
$$


In the presence of a nonuniform exponential trichotomy we consider the subspaces

$$
E(t)=P(t) X, \quad F_{1}(t)=Q_{1}(t) X, \quad \text { and } \quad F_{2}(t)=Q_{2}(t) X .
$$

Let $X$ be the space of continuous functions

$$
\varphi=\left(\varphi_{1}, \varphi_{2}\right):\{(s, \xi) \in \mathbb{R} \times X: \xi \in E(s)\} \rightarrow X
$$

of class $C^{k}$ in $\xi$ such that for every $s \in \mathbb{R}$ and $\xi, \bar{\xi} \in E(s)$ we have:

1.

$$
\varphi(s, 0)=0, \quad \frac{\partial \varphi}{\partial \xi}(s, 0)=0, \quad \varphi(s, E(s)) \subset F_{1}(s) \oplus F_{2}(s)
$$

2.

and

$$
\left\|\frac{\partial^{j} \varphi}{\partial \xi^{j}}(s, \xi)\right\| \leq 1 \text { for } j=1, \ldots, k,
$$

$$
\left\|\frac{\partial^{k} \varphi}{\partial \xi^{k}}(s, \xi)-\frac{\partial^{k} \varphi}{\partial \xi^{k}}(s, \bar{\xi})\right\| \leq\|\xi-\bar{\xi}\| .
$$

Given a function $\varphi \in \mathcal{X}$ we consider its graph

$$
\mathcal{V}=\{(s, \xi, \varphi(s, \xi)):(s, \xi) \in \mathbb{R} \times E(s)\} \subset \mathbb{R} \times X .
$$

We now present the center manifold theorem. We continue to use the notation $p_{s, \xi}=(s, \xi, \varphi(s, \xi))$, and we set $\alpha=4 c D \delta$. We also denote by $\Psi_{\tau}$ the flow generated by the autonomous equation in (32).

Theorem 6.2 ([14, Theorem 3]). If equation (7) admits a nonuniform exponential trichotomy with

$$
(k+1)(a+\varepsilon)-b<0 \text { and }(k+1)(c+\varepsilon)-d<0,
$$

and $\delta$ is sufficiently small, then there is a unique function $\varphi \in \mathcal{X}$ such that the set $\mathcal{V}$ in (50) is invariant under the flow $\Psi_{\tau}$, i.e.,

$$
\Psi_{\tau}\left(p_{s, \xi}\right) \in \mathcal{V} \text { for every }(s, \xi) \in \mathbb{R} \times E(s) \text { and } \tau \in \mathbb{R} .
$$

Furthermore:

1. $\mathcal{V}$ is a smooth manifold of class $C^{k}$ containing the line $\mathbb{R} \times\{0\}$ and satisfying $T_{(s, 0)} \mathcal{V}=\mathbb{R} \times E(s)$ for every $s \in \mathbb{R} ;$

2. there exists $K>0$ such that for each $s \in \mathbb{R}, \xi, \bar{\xi} \in E(s), \tau \in \mathbb{R}$, and $j=0, \ldots, k$ we have

$$
\left\|\frac{\partial^{j} \Psi_{\tau}}{\partial \xi^{j}}\left(p_{s, \xi}\right)-\frac{\partial_{\xi}^{j} \Psi_{\tau}}{\partial \xi^{j}}\left(p_{s, \bar{\xi}}\right)\right\| \leq K e^{(j+1)[(a+\alpha) \tau+\varepsilon|s|]}\|\xi-\bar{\xi}\|, \quad \tau \geq 0,
$$




$$
\begin{aligned}
& \text { and } \\
& \left\|\frac{\partial^{j} \Psi_{\tau}}{\partial \xi^{j}}\left(p_{s, \xi}\right)-\frac{\partial_{\xi}^{j} \Psi_{\tau}}{\partial \xi^{j}}\left(p_{s, \bar{\xi}}\right)\right\| \leq K e^{(j+1)[(c+\alpha)|\tau|+\varepsilon|s|]}\|\xi-\bar{\xi}\|, \quad \tau \leq 0 .
\end{aligned}
$$

The proof of Theorem 6.2 follows a similar strategy to the one described in Section 4.3 in the case of stable manifolds, that is based in a lemma of Henry and in the Faà di Bruno formula to estimate the derivatives of a composition.

The study of center manifolds can be traced back to the works of Pliss [61] and Kelley [42]. A very detailed exposition in the case of autonomous equations is given in [76], adapting results in [78]. See also [51, 77] for the case of equations in infinite-dimensional spaces. We refer to $[20,23,24,76]$ for more details and further references. We emphasize that all these works consider only the case of uniformly hyperbolic dynamics.

\subsection{Reversibility in center manifolds}

We now show that the (time) reversibility of a given flow descends to the center manifold obtained in Theorem 6.2. We recall that time-reversal symmetries are among the fundamental symmetries in many "physical" systems. In particular, many Hamiltonian systems are reversible (see [43] for several examples).

We first introduce the notion of reversible (nonautonomous) differential equation. Let $X$ be a Banach space, and let $L: \mathbb{R} \times X \rightarrow X$ be a continuous function such that

$$
v^{\prime}=L(t, v)
$$

has unique and global solutions. We also consider a (Fréchet) differentiable map $S: \mathbb{R} \times X \rightarrow X$.

Definition 6.3. We say that equation (51) is reversible with respect to $S$ if

$$
L(-t, S(t, v))+\frac{\partial S}{\partial v}(t, v) L(t, v)=-\frac{\partial S}{\partial t}(t, v)
$$

for every $t \in \mathbb{R}$ and $v \in X$.

We now describe a characterization of reversibility. In fact, this characterization can be seen as the main justification for the above notion of reversible equation. For each $s \in \mathbb{R}$ and $v_{s} \in X$, we denote by $\Phi(t, s)\left(v_{s}\right)$ the unique solution of (51) with $v(s)=v_{s}$. We recall that by hypothesis the operator $\Phi(t, s)$ is defined for every $t, s \in \mathbb{R}$.

Theorem 6.4 ([13, Proposition 1]). Equation (51) is reversible with respect to the map $S$ if and only if

$$
\Phi(\tau,-t)(S(t, v))=S(-\tau, \Phi(-\tau, t)(v))
$$


for every $t, \tau \in \mathbb{R}$ and $v \in X$.

We now formulate our result about the reversibility in center manifolds, showing that the reversibility of a given equation, with respect to a map $S$ with $S_{t}=S(t, \cdot)$ linear for each $t \in \mathbb{R}$, always descends to the center manifold. We assume that for some constants $C>0$ and $\theta \geq 0$ we have

$$
C^{-1} e^{-\theta|t|} \leq\left\|S_{t}^{-1}\right\|^{-1} \leq\left\|S_{t}\right\| \leq C e^{\theta|t|}, \quad t \in \mathbb{R} .
$$

We also write

$$
\mathcal{V}_{s}=\{v \in X:(s, v) \in \mathcal{V}\}=\{(\xi, \varphi(s, \xi)): \xi \in E(s)\},
$$

where $\varphi$ is the function given by Theorem 6.2.

Theorem 6.5 ([13, Theorem 2]). Under the assumptions of Theorem 6.2, if the equation

$$
v^{\prime}=A(t) v+f(t, v)
$$

is reversible with respect to a map $S$ with $S_{0}^{2}=\mathrm{Id}$ and $S_{t}$ linear for each $t \in \mathbb{R}$, and the constants in (49) and (52) satisfy

$$
\max \{c, a\}+2(\varepsilon+\theta)<\min \{b, d\},
$$

then $S_{s}\left(\mathcal{V}_{s}\right)=\mathcal{V}_{-s}$ for every $s \in \mathbb{R}$.

\section{Lyapunov regularity}

We present briefly in this section the abstract theory of Lyapunov exponents, and its associated regularity theory developed by Lyapunov in his doctoral thesis [47]. We also explain how one can use this theory to estimate the constants in the notion of nonuniform exponential dichotomy. At present this theory is only fully developed for finite-dimensional spaces. A generalization to infinite dimension is given in [6] in the particular case of Hilbert spaces.

\subsection{Lyapunov exponents and regularity}

Consider matrices $A(t)$ varying continuously with $t \geq 0$. We assume that

$$
\limsup _{t \rightarrow+\infty} \frac{1}{t} \log ^{+}\|A(t)\|=0
$$

where $\log ^{+} x=\max \{0, \log x\}$ (we use the convention that $\log 0=-\infty$ ).

Definition 7.1. We define the Lyapunov exponent $\lambda: \mathbb{R}^{n} \rightarrow \mathbb{R} \cup\{-\infty\}$ associated to equation (7) by

$$
\lambda\left(v_{0}\right)=\limsup _{t \rightarrow+\infty} \frac{1}{t} \log \|v(t)\|,
$$

where $v(t)$ is the solution of $(7)$ with $v(0)=v_{0}$. 
One can easily show that the following properties hold (see [1] for details):

1. $\lambda(\alpha v)=\lambda(v)$ for each $v \in \mathbb{R}^{n}$ and $\alpha \in \mathbb{R} \backslash\{0\}$;

2. for each $v, w \in \mathbb{R}^{n}$ we have

$$
\lambda(v+w) \leq \max \{\lambda(v), \lambda(w)\}
$$

3. for each $v, w \in \mathbb{R}^{n}$ with $\lambda(v) \neq \lambda(w)$ we have

$$
\lambda(v+w)=\max \{\lambda(v), \lambda(w)\} ;
$$

4. given $v_{1}, \ldots, v_{m} \in \mathbb{R}^{n} \backslash\{0\}$, if the numbers $\lambda\left(v_{1}\right), \ldots, \lambda\left(v_{m}\right)$ are distinct, then the vectors $v_{1}, \ldots, v_{m}$ are linearly independent.

By the last property, the function $\lambda$ takes at most $p \leq n$ distinct values on $\mathbb{R}^{n} \backslash\{0\}$, say

$$
-\infty \leq \lambda_{1}<\cdots<\lambda_{p}
$$

Moreover, by the first two properties, for $i=1, \ldots, p$ the set

$$
E_{i}=\left\{v_{0} \in \mathbb{R}^{n}: \lambda\left(v_{0}\right) \leq \lambda_{i}\right\}
$$

is a linear subspace. Note that $\lambda\left(v_{0}\right)>\lambda_{i}$ for every $v_{0} \in \mathbb{R}^{n} \backslash E_{i}$.

To introduce the notion of Lyapunov regularity we consider the initial value problem of the adjoint equation

$$
w^{\prime}=-A(t)^{*} w, \quad w(0)=w_{0}
$$

with $w_{0} \in \mathbb{R}^{n}$, where $A(t)^{*}$ denotes the transpose of $A(t)$. We also consider the corresponding Lyapunov exponent $\mu: \mathbb{R}^{n} \rightarrow \mathbb{R} \cup\{-\infty\}$ defined by

$$
\mu\left(w_{0}\right)=\limsup _{t \rightarrow+\infty} \frac{1}{t} \log \|w(t)\|
$$

where $w(t)$ is the solution of $(54)$.

We denote by $\langle\cdot, \cdot\rangle$ the canonical inner product in $\mathbb{R}^{n}$. We recall that two bases $v_{1}, \ldots, v_{n}$ and $w_{1}, \ldots, w_{n}$ of $\mathbb{R}^{n}$ are said to be dual if $\left\langle v_{i}, w_{j}\right\rangle=\delta_{i j}$ for every $i$ and $j$, where $\delta_{i j}$ is the Kronecker symbol.

Definition 7.2. The regularity coefficient of the pair of Lyapunov exponents $\lambda$ and $\mu$ is defined by

$$
\gamma(\lambda, \mu)=\min \max \left\{\lambda\left(v_{i}\right)+\mu\left(w_{i}\right): 1 \leq i \leq n\right\},
$$

where the minimum is taken over all dual bases $v_{1}, \ldots, v_{n}$ and $w_{1}, \ldots, w_{n}$ of $\mathbb{R}^{n}$. We say that equation (7) is Lyapunov regular or simply regular if $\gamma(\lambda, \mu)=0$.

We note that the notion of regularity can also be expressed solely using equation (7), thus without the need for the adjoint equation in (54). 
Namely, (7) is regular if and only if

$$
\lim _{t \rightarrow+\infty} \frac{1}{t} \int_{0}^{t} \operatorname{tr} A(\tau) d \tau=\sum_{i=1}^{p}\left(\operatorname{dim} E_{i}-\operatorname{dim} E_{i-1}\right) \lambda_{i},
$$

setting $E_{0}=\{0\}$ (we refer to [1] for details). However, it is sometimes convenient to use the above description of regularity, in terms of $\gamma(\lambda, \mu)$, for example in the proof of Theorem 7.3.

\subsection{Existence of nonuniform exponential dichotomies}

We assume in this section that there is at least one negative Lyapunov exponent for equation (7), i.e., that for some $1 \leq k \leq p$ we have

$$
-\infty \leq \lambda_{1}<\cdots<\lambda_{k}<0 \leq \lambda_{k+1}<\cdots<\lambda_{p} .
$$

We emphasize that $\lambda_{k+1}$ may be zero. Furthermore, we assume that there is a subspace $F \subset \mathbb{R}^{n}$ such that $E=E_{k}$ (see (53)) and $F$ give a decomposition

$$
\mathbb{R}^{n}=E \oplus F
$$

with respect to which $A(t)$ has the form

$$
A(t)=\left(\begin{array}{cc}
B(t) & 0 \\
0 & C(t)
\end{array}\right)
$$

for every $t \geq 0$. We also consider the Lyapunov exponents associated to the blocks $B(t)$ and $C(t)$ in (55), i.e., associated to the pair of equations

$$
x^{\prime}=B(t) x \quad \text { and } \quad x^{\prime}=-B(t)^{*} x,
$$

and to the pair of equations

$$
y^{\prime}=C(t) y \quad \text { and } \quad y^{\prime}=-C(t)^{*} y .
$$

The corresponding regularity coefficients are

$$
\gamma_{1}=\gamma(\lambda|E, \mu| E) \quad \text { and } \quad \gamma_{2}=\gamma(\lambda|F, \mu| F) .
$$

The following result shows that any linear differential equation as above admits a nonuniform exponential dichotomy, and in fact a strong nonuniform exponential dichotomy. Furthermore, the constants in the notion of dichotomy can be related to the Lyapunov exponents and the regularity coefficients.

Theorem 7.3 ([12, Theorem 4]). Assume that the matrices $A(t)$ have the form in (55) for every $t \geq 0$, and that equation (7) has at least one negative Lyapunov exponent. Then, for each $\delta>0$, equation (7) admits a strong nonuniform exponential dichotomy with

$$
\begin{aligned}
& \underline{a}=\lambda_{1}+\delta, \quad \bar{a}=\lambda_{k}+\delta, \quad \underline{b}=\lambda_{k+1}+\delta, \quad \bar{b}=\lambda_{p}+\delta, \\
& \text { and } \varepsilon=\max \left\{\gamma_{1}, \gamma_{2}\right\}+2 \delta .
\end{aligned}
$$


By Theorem 7.3, the deviation of a nonuniform exponential dichotomy with respect to a uniform dichotomy is measured by the regularity coefficients in (56). It is therefore of interest to obtain sharp bounds for $\gamma(\lambda, \mu)$, if possible expressed only in terms of the matrices $A(t)$, thus without knowing explicitly the solutions of equation (7).

We first present a lower bound for the regularity coefficient.

Theorem 7.4 ([12, Theorem 5]). We have

$$
\gamma(\lambda, \mu) \geq \frac{1}{n}\left(\limsup _{t \rightarrow+\infty} \frac{1}{t} \int_{0}^{t} \operatorname{tr} A(\tau) d \tau-\liminf _{t \rightarrow+\infty} \frac{1}{t} \int_{0}^{t} \operatorname{tr} A(\tau) d \tau\right) .
$$

We now describe a geometric consequence of Theorem 7.4. Let $v_{1}, \ldots$, $v_{n}$ be a basis of $\mathbb{R}^{n}$. We denote by $\Gamma_{n}(t)$ the $n$-volume of the parallelepiped defined by the vectors $v_{1}(t), \ldots, v_{n}(t)$, where $v_{i}(t)$ is the solution of $(7)$ with $v_{0}=v_{i}$, for $i=1, \ldots, n$. By the Liouville formula we have

$$
\Gamma_{n}(t) / \Gamma_{n}(0)=\exp \int_{0}^{t} \operatorname{tr} A(\tau) d \tau,
$$

and it follows from Theorem 7.4 that

$$
\limsup _{t \rightarrow+\infty} \frac{1}{t} \log \Gamma_{n}(t)-\liminf _{t \rightarrow+\infty} \frac{1}{t} \log \Gamma_{n}(t) \leq n \gamma(\lambda, \mu) .
$$

In particular, when equation (7) is regular we have

$$
\lim _{t \rightarrow+\infty} \frac{1}{t} \log \Gamma_{n}(t)=\lim _{t \rightarrow+\infty} \frac{1}{t} \int_{0}^{t} \operatorname{tr} A(\tau) d \tau
$$

(and the limits are independent of the basis $v_{1}, \ldots, v_{n}$ ).

We now present an upper bound for the regularity coefficient. We only consider triangular matrices, for which we can describe the results more explicitly (we refer to [12] for details on the reduction to the triangular case). For $k=1, \ldots, n$, we consider the numbers

$$
\underline{\alpha}_{k}=\liminf _{t \rightarrow+\infty} \frac{1}{t} \int_{0}^{t} a_{k}(\tau) d \tau \quad \text { and } \quad \bar{\alpha}_{k}=\limsup _{t \rightarrow+\infty} \frac{1}{t} \int_{0}^{t} a_{k}(\tau) d \tau,
$$

where $a_{1}(t), \ldots, a_{n}(t)$ are the entries in the diagonal of $A(t)$.

Theorem 7.5 ([12, Theorem 6$])$. If the matrices $A(t)$ are upper triangular for every $t \geq 0$, then

$$
\gamma(\lambda, \mu) \leq \sum_{k=1}^{n}\left(\bar{\alpha}_{k}-\underline{\alpha}_{k}\right)
$$


It follows from Theorems 7.4 and 7.5 that in the 1-dimensional case, i.e., for a scalar equation $v^{\prime}=a(t) v$ the regularity coefficient is given by

$$
\gamma(\lambda, \mu)=\limsup _{t \rightarrow+\infty} \frac{1}{t} \int_{0}^{t} a(\tau) d \tau-\liminf _{t \rightarrow+\infty} \frac{1}{t} \int_{0}^{t} a(\tau) d \tau
$$

\section{References}

[1] L. Barreira and Ya. Pesin, Lyapunov Exponents and Smooth Ergodic Theory, Univ. Lect. Series 23, Amer. Math. Soc., 2002.

[2] L. Barreira and Ya. Pesin, Smooth ergodic theory and nonuniformly hyperbolic dynamics, with appendix by O. Sarig, in Handbook of Dynamical Systems 1B, edited by B. Hasselblatt and A. Katok, Elsevier, 2006, pp. 57-263.

[3] L. Barreira and Ya. Pesin, Nonuniform Hyperbolicity: Dynamics of Systems with Nonzero Lyapunov Exponents, Encyclopedia of Math. and Its Appl. 115, Cambridge Univ. Press, 2007.

[4] L. Barreira and C. Valls, Higher regularity of invariant manifolds for nonautonomous equations, Nonlinearity 18 (2005), 2373-2390.

[5] L. Barreira and C. Valls, Smoothness of invariant manifolds for nonautonomous equations, Comm. Math. Phys. 259 (2005), 639-677.

[6] L. Barreira and C. Valls, Stability of nonautonomous differential equations in Hilbert spaces, J. Differential Equations 217 (2005), 204-248.

[7] L. Barreira and C. Valls, Existence of stable manifolds for nonuniformly hyperbolic $C^{1}$ dynamics, Discrete Contin. Dyn. Syst. 16 (2006), 307-327.

[8] L. Barreira and C. Valls, A Grobman-Hartman theorem for nonuniformly hyperbolic dynamics, J. Differential Equations 228 (2006), 285-310.

[9] L. Barreira and C. Valls, Smooth invariant manifolds in Banach spaces with nonuniform exponential dichotomy, J. Funct. Anal. 238 (2006), 118-148.

[10] L. Barreira and C. Valls, Stable manifolds for nonautonomous equations without exponential dichotomy, J. Differential Equations 221 (2006), 58-90.

[11] L. Barreira and C. Valls, Hölder Grobman-Hartman linearization, Discrete Contin. Dyn. Syst. 18 (2007), 187-197.

[12] L. Barreira and C. Valls, Nonuniform exponential dichotomies and Lyapunov regularity, J. Dynam. Differential Equations 19 (2007), 215-241.

[13] L. Barreira and C. Valls, Reversibility and equivariance in center manifolds, Discrete Contin. Dyn. Syst. 18 (2007), 677-699.

[14] L. Barreira and C. Valls, Smooth center manifolds for nonuniformly partially hyperbolic trajectories, J. Differential Equations 237 (2007), 307-342.

[15] L. Barreira and C. Valls, Conjugacies for linear and nonlinear perturbtaions of nonuniform behavior, J. Funct. Anal., to appear.

[16] L. Barreira and C. Valls, Optimal estimates along stable manifolds of nonuniformly hyperbolic dynamics, Proc. Roy. Soc. Edinburgh Sect. A, to appear.

[17] L. Barreira and C. Valls, Characterization of stable manifolds for nonuniform exponential dichotomies, preprint.

[18] L. Barreira and C. Valls, Robustness of nonuniform exponential dichotomies in Banach spaces, preprint.

[19] G. Belitski, On the Grobman-Hartman theorem in the class $C^{\alpha}$, preprint.

[20] C. Chicone and Yu. Latushkin, Center manifolds for infinite dimensional nonautonomous differential equations, J. Differential Equations 141 (1997), 356-399. 
[21] C. Chicone and Yu. Latushkin, Evolution Semigroups in Dynamical Systems and Differential Equations, Math. Surveys and Monographs 70, Amer. Math. Soc., 1999.

[22] S.-N. Chow and H. Leiva, Existence and roughness of the exponential dichotomy for skew-product semiflow in Banach spaces, J. Differential Equations 120 (1995), 429-477.

[23] S.-N. Chow, W. Liu and Y. Yi, Center manifolds for invariant sets, J. Differential Equations 168 (2000), 355-385.

[24] S.-N. Chow, W. Liu and Y. Yi, Center manifolds for smooth invariant manifolds, Trans. Amer. Math. Soc. 352 (2000), 5179-5211.

[25] C. Constantine and T. Savits, A multivariate Faà di Bruno formula with applications, Trans. Amer. Math. Soc. 348 (1996), 503-520.

[26] W. Coppel, Dichotomies and reducibility, J. Differential Equations 3 (1967), 500-521.

[27] W. Coppel, Dichotomies in Stability Theory, Lect. Notes in Math. 629, Springer, 1978.

[28] Ju. Dalec'kiı and M. KreĬn, Stability of Solutions of Differential Equations in Banach Space, Translations of Math. Monographs 43, Amer. Math. Soc., 1974.

[29] M. Elbialy, On sequences of $C_{b}^{k, \delta}$ maps which converge in the uniform $C^{0}$-norm, Proc. Amer. Math. Soc. 128 (2000), 3285-3290.

[30] C. Faà di Bruno, Note sur une nouvelle formule du calcul differentiel, Quart. J. Math. 1 (1855), 359-360.

[31] A. Fathi, M. Herman and J.-C. Yoccoz, A proof of Pesin's stable manifold theorem, in Geometric Dynamics (Rio de Janeiro, 1981), edited by J. Palis, Lect. Notes. in Math. 1007, Springer, 1983, pp. 177-215.

[32] D. Grobman, Homeomorphism of systems of differential equations, Dokl. Akad. Nauk SSSR 128 (1959), 880-881.

[33] D. Grobman, Topological classification of neighborhoods of a singularity in $n$ space, Mat. Sb. (N.S.) 56 (98) (1962), 77-94.

[34] M. Guysinsky, B. Hasselblatt and V. Rayskin, Differentiability of the HartmanGrobman linearization, Discrete Contin. Dyn. Syst. 9 (2003), 979-984.

[35] J. Hale, Asymptotic Behavior of Dissipative Systems, Math. Surveys and Monographs 25, Amer. Math. Soc., 1988.

[36] P. Hartman, A lemma in the theory of structural stability of differential equations, Proc. Amer. Math. Soc. 11 (1960), 610-620.

[37] P. Hartman, On the local linearization of differential equations, Proc. Amer. Math. Soc. 14 (1963), 568-573.

[38] D. Henry, Geometric Theory of Semilinear Parabolic Equations, Lect. Notes in Math. 840, Springer, 1981.

[39] P. Hartman, Exponential dichotomies, the shadowing lemma and homoclinic orbits in Banach spaces, in Dynamical Phase Transitions (São Paulo, 1994), Resenhas IME-USP 1 (1994), 381-401.

[40] A. Katok, Lyapunov exponents, entropy and periodic orbits for diffeomorphisms, Inst. Hautes Études Sci. Publ. Math. 51 (1980), 137-173.

[41] A. Katok and B. Hasselblatt, Introduction to the Modern Theory of Dynamical Systems, with a supplement by A. Katok and L. Mendoza, Encyclopedia of Mathematics and its Applications 54, Cambridge University Press, Cambridge, 1995.

[42] A. Kelley, The stable, center-stable, center, center-unstable, unstable manifolds, J. Differential Equations 3 (1967), 546-570. 
[43] J. Lamb and J. Roberts, Time-reversal symmetry in dynamical systems: a survey, in Time-Reversal Symmetry in Dynamical Systems (Coventry, 1996), Phys. D 112 (1998), 1-39.

[44] O. Lanford III, Bifurcation of periodic solutions into invariant tori: the work of Ruelle and Takens, in Nonlinear Problems in the Physical Sciences and Biology: Proceedings of a Battelle Summer Institute (Seattle, 1972), edited by I. Stakgold, D. Joseph and D. Sattinger, Lect. Notes in Math. 322, Springer, 1973, pp. 159192.

[45] F. Ledrappier and L.-S. Young, The metric entropy of diffeomorphisms I. Characterization of measures satisfying Pesin's entropy formula, Ann. of Math. (2) 122 (1985), 509-539.

[46] E. Lukacs, Applications of Faà di Bruno's formula in mathematical statistics, Amer. Math. Monthly 62 (1955), 340-348.

[47] A. Lyapunov, The General Problem of the Stability of Motion, Taylor \& Francis, 1992.

[48] R. Mañé, Lyapunov exponents and stable manifolds for compact transformations, in Geometric dynamics (Rio de Janeiro, 1981), edited by J. Palis, Lect. Notes in Math. 1007, Springer, 1983, pp. 522-577.

[49] J. Massera and J. Schäffer, Linear differential equations and functional analysis. I, Ann. of Math. (2) 67 (1958), 517-573.

[50] J. Massera and J. Schäffer, Linear Differential Equations and Function Spaces, Pure and Applied Mathematics 21, Academic Press, 1966.

[51] A. Mielke, A reduction principle for nonautonomous systems in infinitedimensional spaces, J. Differential Equations 65 (1986), 68-88.

[52] J. Moser, On a theorem of Anosov, J. Differential Equations 5 (1969), 411-440.

[53] R. Naulin and M. Pinto, Admissible perturbations of exponential dichotomy roughness, Nonlinear Anal. 31 (1998), 559-571.

[54] V. Oseledets, A multiplicative ergodic theorem. Liapunov characteristic numbers for dynamical systems, Trans. Moscow Math. Soc. 19 (1968), 197-221.

[55] J. Palis, On the local structure of hyperbolic points in Banach spaces, An. Acad. Brasil. Ci. 40 (1968), 263-266.

[56] K. Palmer, A generalization of Hartman's linearization theorem, J. Math. Anal. Appl. 41 (1973), 753-758.

[57] O. Perron, Die Stabilitätsfrage bei Differentialgleichungen, Math. Z. 32 (1930), 703-728.

[58] Ya. Pesin, Families of invariant manifolds corresponding to nonzero characteristic exponents, Math. USSR-Izv. 10 (1976), 1261-1305.

[59] Ya. Pesin, Characteristic Ljapunov exponents, and smooth ergodic theory, Russian Math. Surveys 32 (1977), 55-114.

[60] Ya. Pesin, Geodesic flows on closed Riemannian manifolds without focal points, Math. USSR-Izv. 11 (1977), 1195-1228.

[61] V. Pliss, A reduction principle in the theory of stability of motion, Izv. Akad. Nauk SSSR Ser. Mat. 28 (1964), 1297-1324.

[62] V. Pliss and G. Sell, Robustness of exponential dichotomies in infinite-dimensional dynamical systems, J. Dynam. Differential Equations 11 (1999), 471-513.

[63] L. Popescu, Exponential dichotomy roughness on Banach spaces, J. Math. Anal. Appl. 314 (2006), 436-454.

[64] C. Pugh, On a theorem of P. Hartman, Amer. J. Math. 91 (1969), 363-367.

[65] C. Pugh, The $C^{1+\alpha}$ hypothesis in Pesin theory, Inst. Hautes Études Sci. Publ. Math. 59 (1984), 143-161. 
[66] C. Pugh and M. Shub, Ergodic attractors, Trans. Amer. Math. Soc. 312 (1989), $1-54$.

[67] V. Rayskin, $\alpha$-Hölder linearization, J. Differential Equations 147(1998), 271-284.

[68] D. Ruelle, Ergodic theory of differentiable dynamical systems, Inst. Hautes Études Sci. Publ. Math. 50 (1979), 27-58.

[69] D. Ruelle, Characteristic exponents and invariant manifolds in Hilbert space, Ann. of Math. (2) 115 (1982), 243-290.

[70] R. Sacker, Existence of dichotomies and invariant splittings for linear differential systems IV, J. Differential Equations 27 (1978), 106-137.

[71] R. Sacker and G. Sell, Existence of dichotomies and invariant splittings for linear differential systems I, J. Differential Equations 15 (1974), 429-458.

[72] R. Sacker and G. Sell, Existence of dichotomies and invariant splittings for linear differential systems II, J. Differential Equations 22 (1976), 478-496.

[73] R. Sacker and G. Sell, Existence of dichotomies and invariant splittings for linear differential systems III, J. Differential Equations 22 (1976), 497-522.

[74] R. Sacker and G. Sell, Dichotomies for linear evolutionary equations in Banach spaces, J. Differential Equations 113 (1994), 17-67.

[75] G. Sell and Y. You, Dynamics of Evolutionary Equations, Applied Math. Sciences 143, Springer, 2002.

[76] A. Vanderbauwhede, Centre manifolds, normal forms and elementary bifurcations, in Dynamics Reported 2, Wiley, 1989, pp. 89-169.

[77] A. Vanderbauwhede and G. Iooss, Center manifold theory in infinite dimensions, in Dynamics Reported (N.S.) 1, Springer, 1992, pp. 125-163.

[78] A. Vanderbauwhede and S. van Gils, Center manifolds and contractions on a scale of Banach spaces, J. Funct. Anal. 72 (1987), 209-224.

[79] S. van Strien, Smooth linearization of hyperbolic fixed points without resonance conditions, J. Differential Equations 85 (1990), 66-90. 